\title{
Lattice orientation and crack size effect on the mechanical properties of Graphene
}

\author{
P. R. Budarapu • B. Javvaji - V. K. Sutrakar • \\ D. Roy Mahapatra · M. Paggi - G. Zi · T. Rabczuk
}

Received: 26 January 2016 / Accepted: 11 April 2016

(C) Springer Science+Business Media Dordrecht 2016

\begin{abstract}
The effect of lattice orientation and crack length on the mechanical properties of Graphene are studied based on molecular dynamics simulations. Bond breaking and crack initiation in an initial edge crack model with 13 different crack lengths, in 10 different lattice orientations of Graphene are examined. In all the lattice orientations, three recurrent fracture patterns are reported. The influence of the lattice orientation and crack length on yield stress and yield strain of Graphene is also investigated. The arm-chair fracture pattern is observed to possess the lowest yield properties. A sudden decrease in yield stress and yield strain can be noticed for crack sizes $<10 \mathrm{~nm}$. However, for
\end{abstract}

P. R. Budarapu $(\varangle) \cdot$ M. Paggi

IMT School for Advanced Studies Lucca, Piazza San

Fracensco 19, 55100 Lucca, Italy

e-mail: pattabhi.budarapu@imtlucca.it

B. Javvaji · D. Roy Mahapatra

Department of Aerospace Engineering, Indian Institute of

Science, Bangalore 560012, India

\section{K. Sutrakar}

Aeronautical Development Agency, Defence Research and Development Organization, Bangalore 560017, India

G. Zi · T. Rabczuk

School of Civil, Environmental and Architectural

Engineering, Korea University, Seoul 136-701, Korea

T. Rabczuk

Institute of Structural Mechanics, Bauhaus University of Weimar, 99423 Weimar, Germany larger crack sizes, a linear decrease in yield stress is observed, whereas a constant yield strain of $\approx 0.05$ is noticed. Therefore, the yield strain of $\approx 0.05$ can be considered as a critical strain value below which Graphene does not show failure. This information can be utilized as a lower bound for the design of nano-devices for various strain sensor applications. Furthermore, the yield data will be useful while developing the Graphene coating on Silicon surface in order to enhance the mechanical and electrical characteristics of solar cells and to arrest the growth of micro-cracks in Silicon cells.

Keywords Graphene fracture - Molecular dynamics . Bond elongation and rotation - Lattice orientation and initial crack size

\section{Introduction}

Graphene, an ultimately thin monolayer of carbon atoms packed into a hexagonal lattice, is the basic building block for graphitic materials of all other dimensionalities (Geim 2009; Huhu et al. 2014; Morpurgo 2015; Ying-Yan et al. 2014). Because of its several useful material properties (Changgu et al. 2008; Fengnian et al. 2014; Fiori et al. 2014; Kravets et al. 2014; Mics et al. 2015; Sarma et al. 2011; Schwierz 2010; Weiwei et al. 2015), Graphene finds wide range of applications (Budarapu et al. 2009, 2014c; Kinam et al. 2011; Kostarelos and Novoselov 2014; Liu 2014; Pospischil et al. 2014; Quan et al. 2015; Rodrigues et al. 2015; San- 
tanu et al. 2012; Son et al. 2015; Traversi et al. 2014; Wen et al. 2012; Xue et al. 2014; Xuechao et al. 2015; Zhang et al. 2013). It can also be used as a strengthening component in composites (Chen et al. 2015a; Kim et al. 2013; Shin et al. 2015; Zan et al. 2015). As the demand for Graphene-based applications is growing, it is important to deeply understand it's mechanical and challenging failure characteristics. Chuvilin et al. (2009) studied the growth of two holes created by radiation in Graphene nano ribbons, based on the imaging side spherical aberration-corrected transmission electron microscopy at $80 \mathrm{kV}$. As the holes grow and two holes approach each other, they observed the transitions and deviations from the hexagonal structure involving some reconstructions resulting into more pentagons and heptagons than hexagons, which turned out to be stable. Jin et al. (2009) explained the structural dynamics of carbon atomic chains such as formation, migration, and breakage observed in the experiments, by density-functional theory calculations. However, estimating the mechanical properties of Graphene is challenging due to the practical difficulties in settingup experiments (Kim et al. 2012). Hence, numerical simulations are good alternatives for predicting fracture related properties. Several researchers have investigated the mechanical properties of Graphene based on molecular dynamics simulations (Chen et al. 2015b; Gamboa et al. 2015; Grantab et al. 2010; Hadden et al. 2015; Pei et al. 2010; Tiwary et al. 2015a, b; Vadukumpully et al. 2011).

Bu et al. (2009) investigated the mechanical behavior of Graphene nanoribbons based on Tersoff potential function. They reported the increase in the Young's modulus due to stress stiffening, when the strain exceeds $18 \%$. Peng et al. (2014) have measured the fracture toughness of Graphene based on experiments, validated with numerical simulations. They also verified the applicability of the classic Griffith theory of brittle fracture to Graphene. They observed that the critical stress intensity factor $\left(\mathrm{K}_{I C}\right)$ is not constant when the initial crack length is less than a certain value. Ansari et al. (2012) have studied the presence of vacancy defects in Graphene based on the Tersoff-Brenner potential function and reported significant reduction in the ultimate strength in the zig-zag direction, while the effect is minimal on the Young's modulus. Khare et al. (2007) have studied the effects of large defects and cracks on the mechanical properties of carbon nanotubes and Graphene sheets using the coupled quantum mechanical/molecular mechanical approach based on the Tersoff-Brenner potential. They observed that the weakening effects of holes, slits, and cracks will vary only moderately with the shape of the defect, and instead depend primarily on the crosssection of the defect perpendicular to the loading direction and on the structure near the fracture initiation point.

Jhon et al. (2012, 2014) estimated the anisotropic fracture response of Graphene based on molecular dynamics simulations. They found that both the tensile strength and strain remain almost constant up to an orientation angle of $12^{\circ}$. Then a rapid increase resulting in a remarkable degradation of the tensile strength compared to brittle fracture counterpart, was reported. They also noticed that fracture pattern holds in the range 100 $700 \mathrm{~K}$. Sun et al. (2015) investigated the orientational anisotropic effect on the fracture strength of vacancydefective Graphene using molecular dynamics simulations. They concluded that the fracture strength of Graphene at the orientation angle of $15^{\circ}$ has the smallest sensitivity to vacancy defects due to the minimization of stress concentration in that direction. Also, the fracture strength in the zig-zag direction was found to be more sensitive to the vacancy defects. Cao (2014) used the quantum mechanical and classical molecular dynamics simulations to understand the mechanical behavior of Graphene. However, the above studies were carried out on Graphene without initial cracks. Hence, the effect of lattice orientation on the crack growth dynamics was ignored.

Zhao et al. (2009) have investigated the mechanical strength and properties of Graphene under uniaxial tensile test as a function of size and chirality using the orthogonal tight-binding method and molecular dynamics simulations with an adaptive intermolecular reactive empirical bond order (AIREBO) potential. They reported reasonable agreement of their estimated results on Young's modulus, fracture strain and fracture strength of bulk Graphene, with the published experimental data (Changgu et al. 2008; Liu et al. 2007). They also observed that the Griffith criterion overestimates the strength of cracks shorter than $10 \mathrm{~nm}$ and hence used a strength based criterion to explain the yield behaviour.

Recently, Datta et al. (2015) have investigated:

1. The effect of two lattice orientations (arm-chair and zig-zag) with different crack lengths (for a/b ratios 
upto 0.25 ) in mixed mode loading conditions at 300 and $1000 \mathrm{~K}$, while keeping the crack perpendicular to the orientations.

2. The effect of crack orientation in the arm-chair and zig-zag Graphene, retaining the same loading directions.

Their main objective is to study the fracture toughness, using the AIREBO potential function. In the present work, we study 10 different orientations with 13 different crack lengths. We always load Graphene along the direction perpendicular to the crack length to study the Mode I fracture. This is similar to the first case investigated in Datta et al. (2015) for arm-chair and zig-zag Graphene. In the present work, our focus is mainly on estimating the mechanical properties of Graphene and the coupled effect of the lattice orientations and initial crack length on the mechanical properties, based on the Tersoff potential function. Therefore, the relations between the lattice orientation and the crack pattern and between the initial crack size and lattice orientation on the mechanical properties of Graphene are investigated. The final aim is to come up with a design criterion which can be used in the nano-devices for strain sensor applications.

The followings are the main objectives of the present study: (1) identification of limiting strain below which Graphene never fails; (2) combined effect of the lattice orientation and crack size on the mechanical properties of Graphene; (3) identification of fracture pattern for each given lattice orientation; and (4) the variation of the tensile strength with chiral angle. All the above results are important for the design of Graphene-based nano devices.

The arrangement of the article is as follows: Details of the numerical model are explained in Sect. 2. The effect of lattice orientation and crack size in the yield properties of Graphene are discussed in Sect. 3. The key findings are summarized in Sect. 4.

\section{Atomistic modelling and simulations}

In this work, the atom to atom interactions of carbon in Graphene are simulated based on the Tersoff potential (1989). Tersoff potential has been successfully applied to predict mechanical properties of Graphene (Bu et al. 2009; Budarapu et al. 2015; Thomas and Ajith 2014; Volokh 2012). The mathematical expression of the bond energy of the atomistic model based on the Tersoff

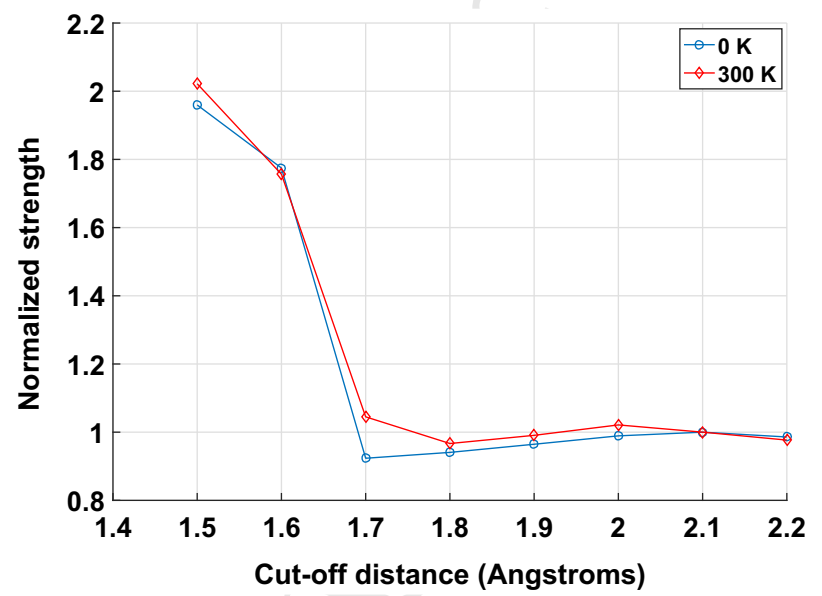

Fig. 1 Variation of the normalized strength at the first bond break with cut-off distance at 0 and $300 \mathrm{~K}$, with an initial crack length of $0.5 \mathrm{~L}$ and when the lattice is oriented along $0^{\circ}$. The fracture strength at the first bond break is normalized with the corresponding value at $\mathrm{r}_{c}=2.1 \AA$

potential can be expressed as (Tersoff 1989):

$V\left(r_{\alpha \beta}\right)=f_{c}\left(r_{\alpha \beta}\right)\left[f_{R}\left(r_{\alpha \beta}\right)+b_{\alpha \beta} f_{A}\left(r_{\alpha \beta}\right)\right]$

$$
f_{R}>f_{c}, \quad f_{A}>b_{\alpha \beta}
$$

where $r_{\alpha \beta}$ is the distance between the atoms $\alpha$ and $\beta$. The bond energy in the Tersoff framework is a combination of attractive $\left(f_{A}\right)$ and repulsive $\left(f_{R}\right)$ energy functions, which are expressed in the form of the exponential Morse like functions; $f_{c}$ is a smooth spherical cut-off function around atom $\alpha$ based upon the distance to the first nearest neighbour cell. Details of the variables of potential function in Eq. (1) are explained in "Appendix". Variation of the normalized fracture strength with the effect of cut-off distance $\left(r_{c}\right)$ at 0 and $300 \mathrm{~K}$ are plotted in Fig. 1. In Fig. 1, the fracture strength at the first bond break is normalized with the corresponding value at $\mathrm{r}_{c}=2.1 \AA$ A. According to Fig. 1 and to (Shenderova et al. 2000; Zhao and Aluru 2010), a cut-off distance of $2.1 \AA$ is considered to reproduce the physical observations, even at higher temperatures. Hence, $r_{c}=2.1 \AA$ is used in all the simulations of the present work.

The main aim of the present work is to understand the effect of lattice orientation on the crack initiation and growth mechanics and hence, on the mechanical properties of Graphene. To achieve this objective, an uni-axial tensile deformation test of Graphene is studied with varying lattice orientation and initial crack length $\left(\mathrm{a}_{0}\right)$. Ten different lattice orientations of Graphene are characterized by the following chiral vec- 


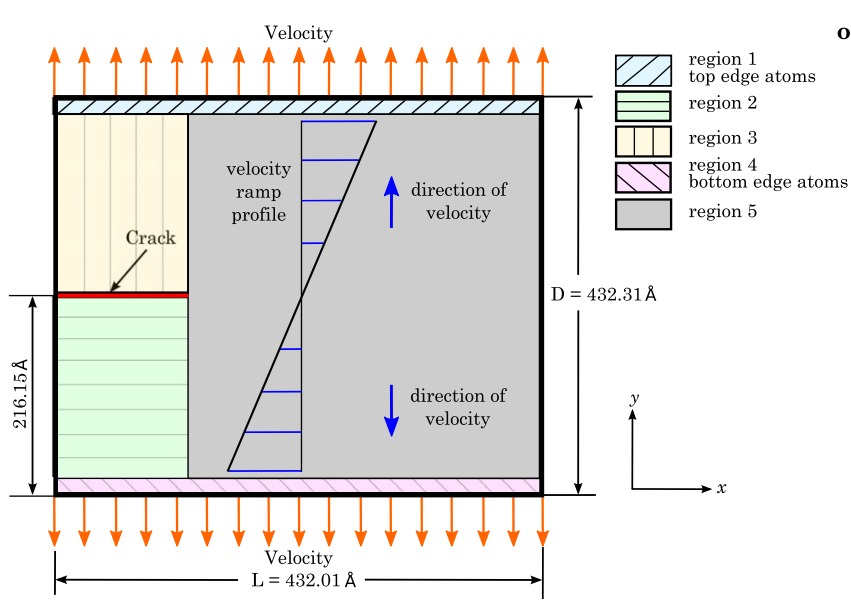

(a)

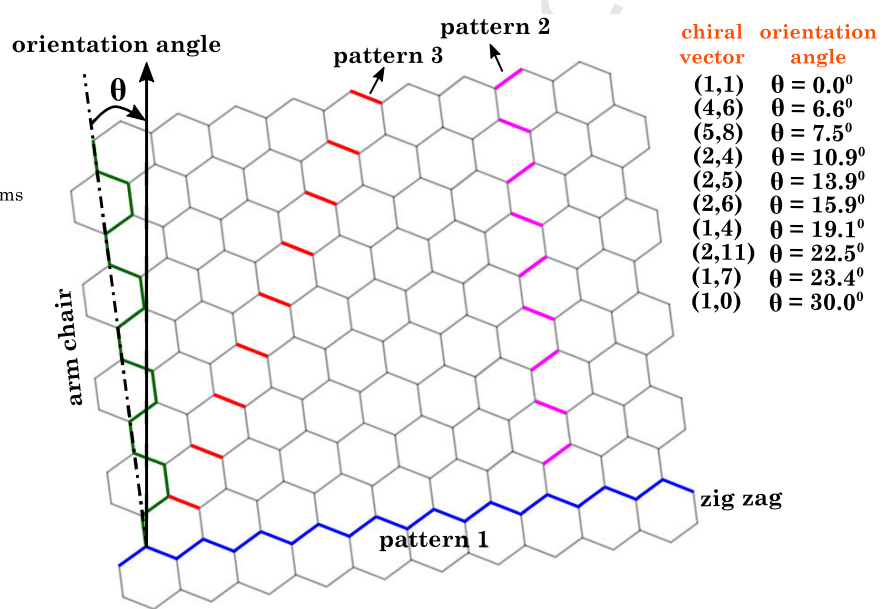

(b)

Fig. 2 a Schematic of the edge crack model used in all the examples and $\mathbf{b}$ schematic showing the observed three fracture patterns along with the orientation angle, arm-chair and zig-zag directions

tors: $(1,0),(1,1),(4,6),(5,8),(2,4),(2,5),(2,6),(1,4)$ $(2,11),(1,7)$. Thirteen different initial crack lengths are also considered, namely: $0.025 \mathrm{~L}, 0.05 \mathrm{~L}, 0.1 \mathrm{~L}, 0.15 \mathrm{~L}$, $0.2 \mathrm{~L}, 0.25 \mathrm{~L}, 0.33 \mathrm{~L}, 0.4 \mathrm{~L}, 0.5 \mathrm{~L}, 0.6 \mathrm{~L}, 0.7 \mathrm{~L}, 0.8 \mathrm{~L}$, and 0.9L, where $\mathrm{L}$ is the width of the sample, see Fig. 2.

The hexagonal lattice structure of the Graphene sheet with a lattice constant $2.45 \AA$, is considered to develop the atomistic model. An initial edge crack is created in the middle of the vertical side. The lattice domain has overall dimensions of 432.01, $\AA$ (L) $\times$ 432.31 $\AA$ (D), as shown in Fig. 2a. The corresponding full scale atomistic model consists of a set of atoms ranging from 74,880 to 75,269 , depending on the lattice orientation angle. To model the crack, the total domain is partitioned into several regions, see Fig. 2a. Atoms on the top and bottom edges of the domain belong to regions 1 and 4, respectively. The crack in the atomistic model is identified based on the distance between two neighboring atoms. However, the initial crack in the atomistic model is created by restricting the interactions between the set of atoms on either side of the crack surface, which is achieved by updating the neighbour list accordingly. The degrees of freedom along the $x$-direction of the left and right edge atoms and along the $y$-direction of the top and bottom edge atoms, are restrained. An initial velocity of 0.1 angstroms/pico-seconds ( $\AA / \mathrm{ps})$ along the $y$-direction is prescribed on the top and bottom edge atoms. A ramp velocity profile as shown in Fig. 2a is adopted for the rest of the domain. All the simulations in the present work are carried out using the open source Large- scale Atomic/Molecular Massively Parallel Simulator (LAMMPS) software (Plimpton 1995).

In the present work, a 'load cycle' is defined as prescribing the velocity on the top and bottom edge atoms for a specified time period, followed by an equilibration for another specified time period. In each load cycle, the prescribed velocity on the top and bottom edge atoms is applied for another period of $1 \mathrm{ps}$, after which the system is equilibrated for a period of $1 \mathrm{ps}$. The computed stress is the averaged stress estimated based on the Virial theorem (Marc and McMillan 1985; Subramaniyan and Sun 2008). The average virial stress $(\boldsymbol{\sigma})$ over a volume $\Omega$ with total number of atoms $n^{A}$ is calculated as

$\boldsymbol{\sigma}=\frac{1}{\Omega}\left(\frac{1}{2} \sum_{\alpha=1}^{n^{A}} \sum_{\substack{\beta=1, \beta \neq \alpha}}^{n^{A}} \mathbf{r}_{\alpha \beta} \otimes \mathbf{f}_{\alpha \beta}-\sum_{\alpha=1}^{n^{A}} m_{\alpha} \dot{\mathbf{u}}_{\alpha} \otimes \dot{\mathbf{u}}_{\alpha}\right)$

where $m_{\alpha}$ is the mass of atom $\alpha, \mathbf{r}$ is the position vector, $\mathbf{f}$ is the force vector and $\mathbf{u}_{\alpha}, \dot{\mathbf{u}}_{\alpha}$ are the displacement and velocity vectors of atom $\alpha$, respectively. The definition of Virial stress involves the instantaneous velocities only due to thermal fluctuation. Therefore, the Virial stress calculated from molecular dynamics simulations has to be time averaged in order to arrive at the equivalent continuum Cauchy stress. In this work, Virial stress is averaged over 500 time steps. Engineering strain is used as a measure of deformation, which is defined as $\left(l-l_{0}\right) / l_{0}$, where $l$ is the instantaneous length of the 
vertical side and $l_{0}$ is its initial value obtained after the first step of energy minimization corresponding to the initial configuration. The time integration of the equations of motion is carried out based on the Verlet algorithm (Swope et al. 1982). The effect of numerical stability has been investigated in Budarapu et al. (2015). Budarapu et al. (2015) have reported that $1.0 \mathrm{fs}$ is sufficient to study the mechanical behavior of Graphene up to yielding. However, a much smaller time step is required to predict the crack growth more accurately. Since the objective is to study the yield properties, a time step of $1.0 \mathrm{fs}$ has been considered in the present work.

All the simulations are performed under isothermal loading conditions, at a temperature of $\approx 0 \mathrm{~K}(0.1 \mathrm{~K})$. In the isothermal loading, the system temperature is maintained constant. However, the application of the initial velocities would lead to increase in the kinetic energy and hence, the system temperature. Therefore, the isothermal conditions in the present work are achieved by velocity rescaling technique, where the velocities at each time step are rescaled to maintain the constant temperature of $\approx 0 \mathrm{~K}$. The temperature of $\approx 0 \mathrm{~K}$ is considered to avoid the influence of temperature on the lattice orientations and crack length in the mechanical behavior of Graphene. Furthermore, to observe the trends in mechanical properties with temperature, some simulations are carried out at $300 \mathrm{~K}$. The stress-strain curves at $\approx 0 \mathrm{~K}$ are compared to the results at $300 \mathrm{~K}$, as explained in the results and discussion Sect. 3. However, the complete study of the effect of temperature on the mechanical response by varying lattice orientations and crack length is beyond the scope of the present work.

The maximum Cauchy stress for a uni-axial tensile test in the arm-chair and zig-zag direction is found to be 110 and $121 \mathrm{GPa}$ (Liu et al. 2007; Zhao et al. 2009), respectively. The intrinsic breaking strength of perfect Graphene is reported as $130 \pm 10 \mathrm{GPa}$ (Changgu et al. 2008; Zhao et al. 2009). Depending on the size of the defect, the fracture stress of the defective Graphene varies from 30 to $120 \mathrm{GPa}$ (Khare et al. 2007; Zhang et al. 2012b). However, the fracture stress is observed to drop sharply from $120 \mathrm{GPa}$, for small initial defect sizes and tend to 30-60 GPa, after a certain defect size. The ability of a material containing a crack to resist fracture is measured by its fracture toughness property. The critical stress intensity factor (Peng et al. 2014) denoted by $\mathrm{K}_{I C}=\sigma_{c} \sqrt{\pi a_{0}}$, where $\sigma_{c}$ is the critical stress of onset of fracture and $a_{0}$ is the initial crack length, is conventionally used to characterize the fracture toughness of Graphene. As reported in Peng et al. (2014), the factor $\sigma_{c} \sqrt{a_{0}}$ remains constant irrespective of the initial crack length. Therefore, the fracture toughness of Graphene is expected to remain constant with respect to the size of the initial crack as well.

\section{Results and discussions}

\subsection{Orientation dependent crack pattern}

We performed the fracture simulations of 10 different lattice orientations with 13 different initial crack lengths, to estimate the yield properties corresponding to each combination of lattice orientation and initial crack length. The yield properties, namely the yield stress and yield strain are the stress and strain values captured at the time of first bond break. Based on the results, three different patterns of crack growth in Graphene as labeled in Fig. 2b are observed. The pattern labeled as 1 is observed in the arm-chair Graphene $\left(0^{\circ}\right)$. A similar pattern is also observed in the $6.6^{\circ}$, and $10.9^{\circ}$ orientations. The second pattern is noticed only in the zig-zag Graphene, oriented at $30^{\circ}$. Whereas, the third pattern is observed when the Graphene is oriented along the remaining six orientations, namely: $7.5^{\circ}, 13.9^{\circ}, 15.9^{\circ}, 19.1^{\circ}, 22.5^{\circ}$, and $23.4^{\circ}$. Therefore, we specifically select $0^{\circ}, 13.9^{\circ}$, and $30^{\circ}$ orientations to understand the crack growth patterns and hence the variation of the associated mechanical properties.

The initial atomic configuration at $0^{\circ}$ orientation is shown in Fig. 3a. Atoms around the crack tip are marked with labels ' $A$ ' to ' $\mathrm{P}$ ', where the color of the atoms indicates their potential energy. A portion of the atoms around the crack tip for the $0^{\circ}, 13.9^{\circ}$ and $30^{\circ}$ lattice orientations is also shown in Figs. 4a, 5a and 6a, respectively. In the initial configuration, all the atoms are assumed to have the same potential energy. The initial crack is created by deleting the bonds between the atoms and updating the neighbour list accordingly. The dashed lines in Figs. 4a, 5a and 6a shows the deleted bonds and hence the size of the initial crack. A strain load is prescribed on a group of atoms along the top and bottom surfaces of the lattice, as shown in Fig. 2a. Therefore, based on the given loading and boundary conditions, the bond $\mathrm{D}-\mathrm{C}$ is the first loaded bond. Con- 
$\boldsymbol{\theta}=\mathbf{0}^{\mathbf{0}}$

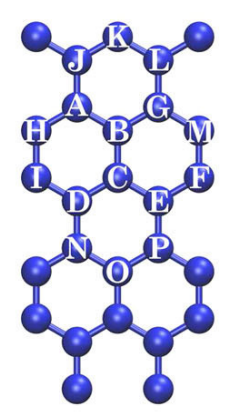

(a) $\mathrm{t}=\mathbf{0 ~ p s}$

$\theta=13.9^{0}$

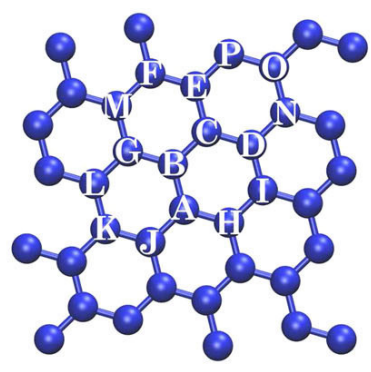

(d) $\mathrm{t}=\mathbf{0} \mathrm{ps}$

$\theta=30^{0}$

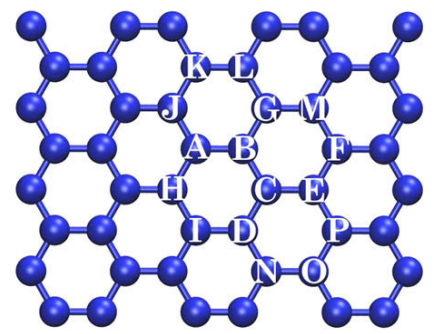

(g) $\mathrm{t}=0 \mathrm{ps}$

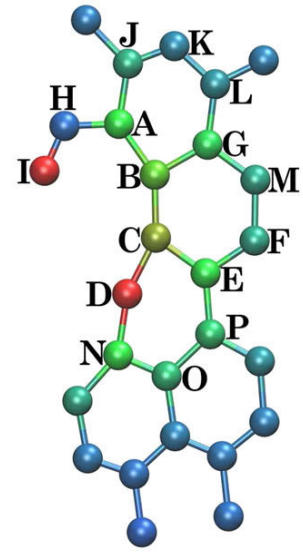

(b) $t=212 \mathrm{ps}$

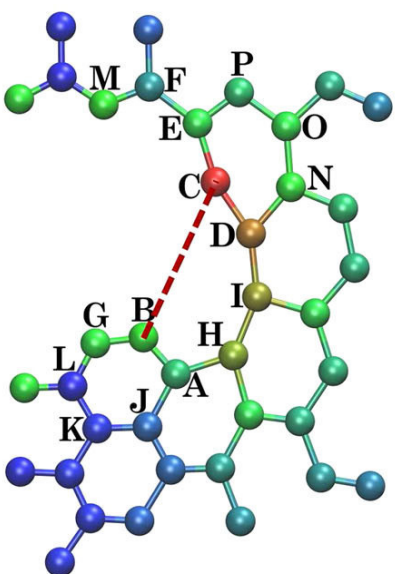

(e) $\mathrm{t}=212.5 \mathrm{ps}$

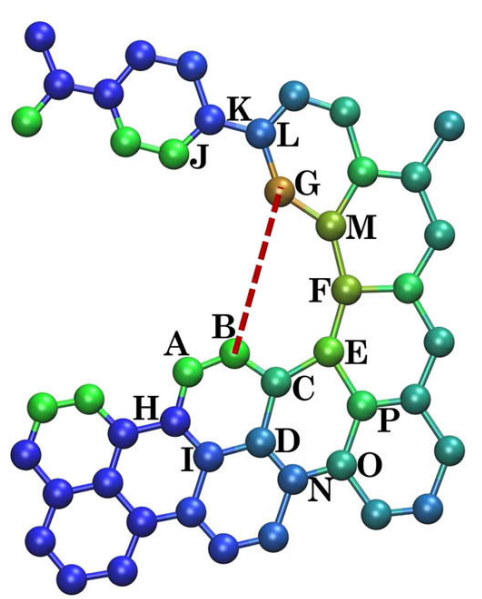

(h) $\mathrm{t}=216 \mathrm{ps}$

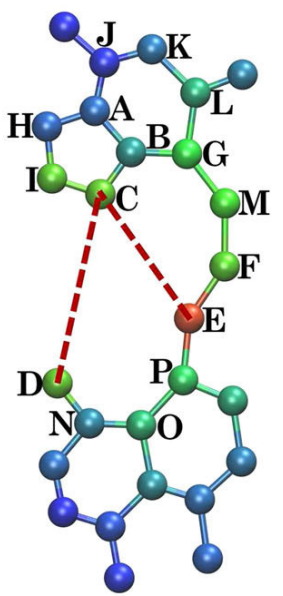

(c) $\mathrm{t}=213.5 \mathrm{ps}$
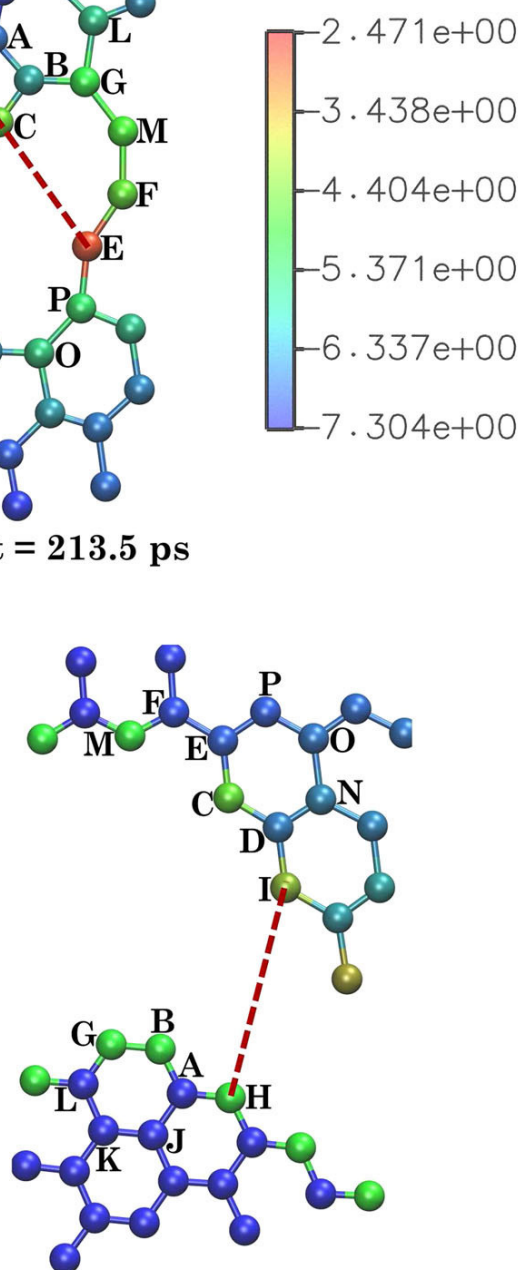

(f) $\mathrm{t}=232 \mathrm{ps}$

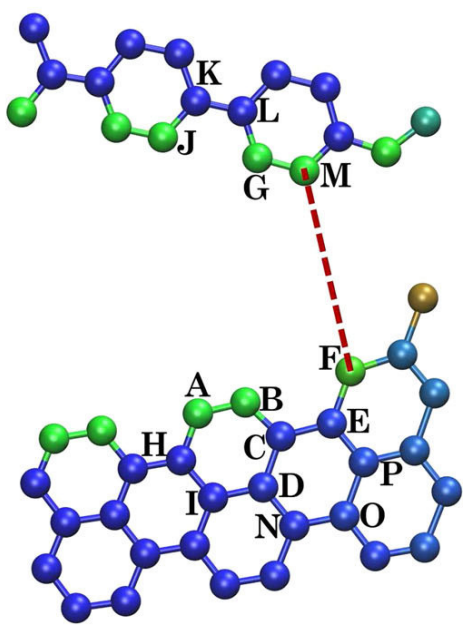

(i) $\mathrm{t}=216.5 \mathrm{ps}$

Fig. 3 Atomic configurations around the crack tip in the initial and during the first and second bond break, when the Graphene lattice is oriented along $0^{\circ}, 13.9^{\circ}$ and $30^{\circ}$, in the top, middle and bottom rows respectively. The dashed lines indicate the broken bonds 


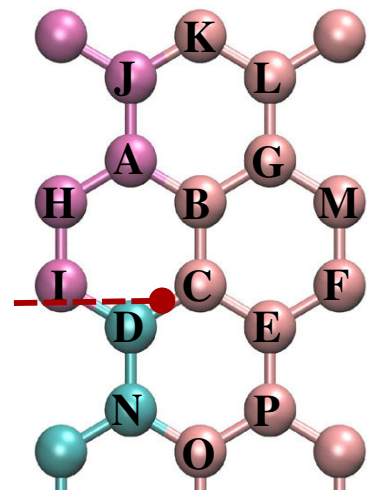

(a) $0^{\circ}$

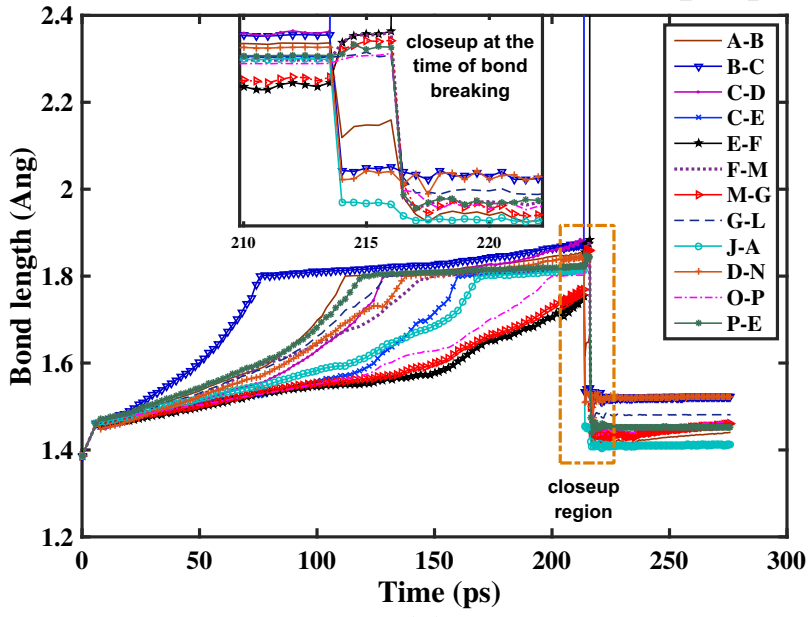

(b)

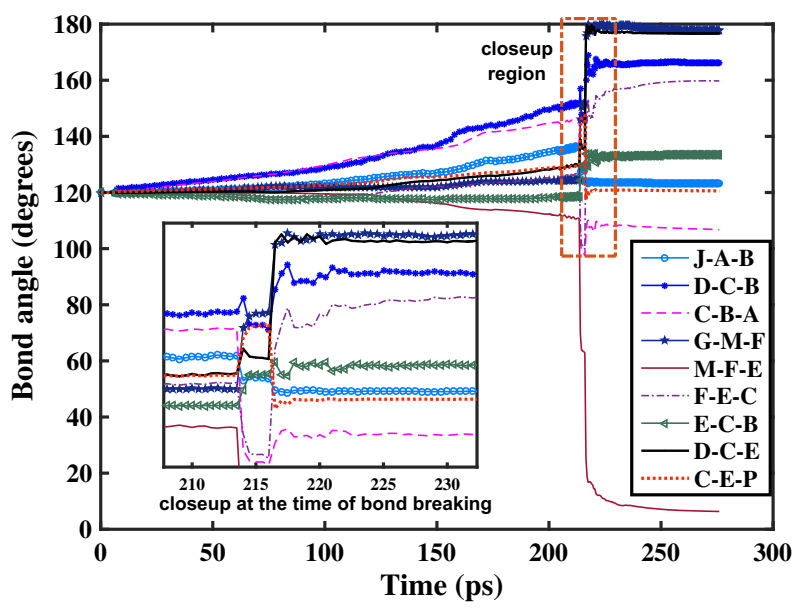

(c)

Fig. 4 a Atom position in the initial configuration when the lattice is oriented along the $0^{\circ}$, along with the atoms around the crack tip. Variation of the $\mathbf{b}$ bond length, $\mathbf{c}$ bond angles and $\mathbf{d}$

tinuous increase loading leads to stretching of the bonds connecting the atoms D-C-E. When the bond length reaches a certain threshold, the bond between D-C breaks first at $213 \mathrm{ps}$, further transferring the load to the next symmetric bond $\mathrm{C}-\mathrm{E}$, which finally breaks at 213.5 ps as shown in Fig. 3c. The load transfer is confirmed by the shifting of the higher energy from atom $D$ to atom E, refer to Fig. 3a-c. The next bond to break is E-F. Remaining bonds like A-B, B-G, D-N and N-O are found to be stable with equilibrium bond length.

The initial configuration with the lattice oriented along the $13.9^{\circ}$ is shown in Fig. 3d. In this orientation, the bond $\mathrm{B}-\mathrm{C}$ is observed to be the first loaded,

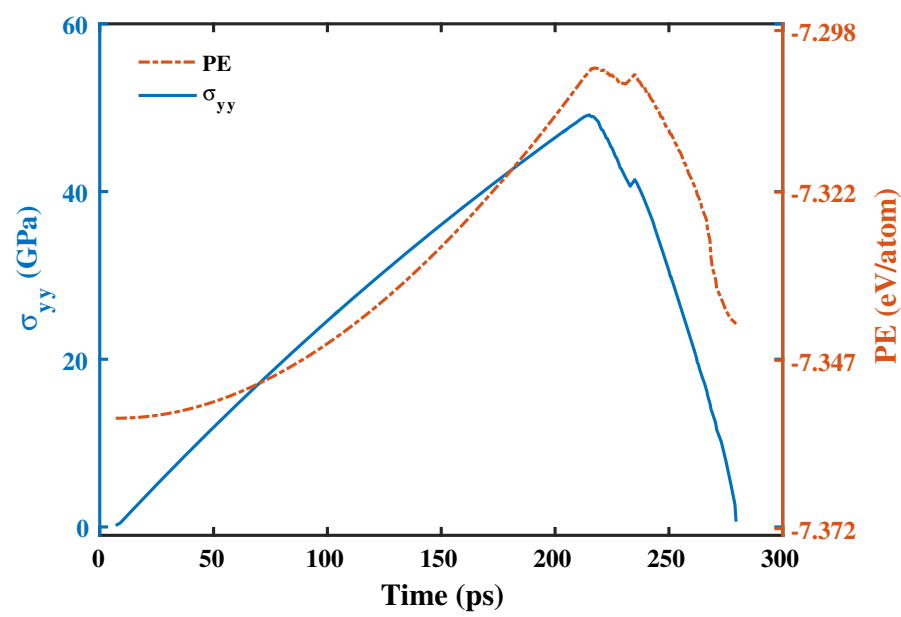

(d)

strain along the $y$ direction and the potential energy per atom, with time. The pictures in the bottom of $\mathbf{c}$ shows a closeup at the time of bond breaking. Plot $\mathbf{d}$ is generated for $\mathrm{a}_{0}=0.5 \mathrm{~L}$

which breaks at $212.5 \mathrm{ps}$ as indicated in Fig. 3e. After the failure of the bond, the load is observed to be transferred to atom $\mathrm{I}$ from atom $\mathrm{C}$ through atom $\mathrm{D}$. This leads to the failure of bond $\mathrm{I}-\mathrm{H}$ at $232 \mathrm{ps}$, see Fig. 3f.

The initial configuration when the lattice is oriented along $30^{\circ}$, is shown in Fig. 3g. In this configuration, the bond connecting atoms $\mathrm{G}-\mathrm{B}$ is observed to break first at $216 \mathrm{ps}$, followed by the failure of the symmetric bond M-F at 216.5 ps, refer to Fig. 3h, i.

Initial configurations of the lattice oriented along $0^{\circ}$, $13.9^{\circ}$ and $30^{\circ}$ are shown in Figs. $4 a$, 5a and 6a, respectively. Stretching of various bonds with time in the $0^{\circ}$ 


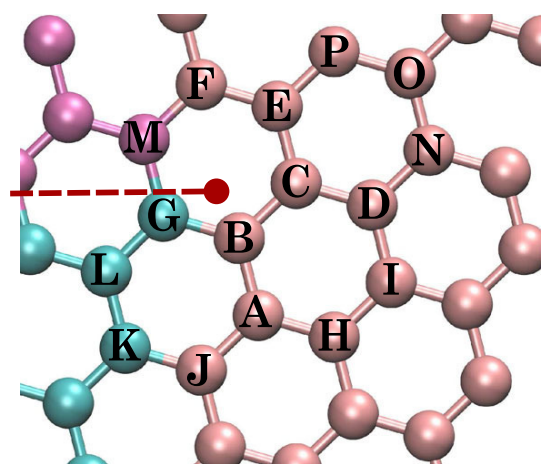

(a) $30^{\circ}$

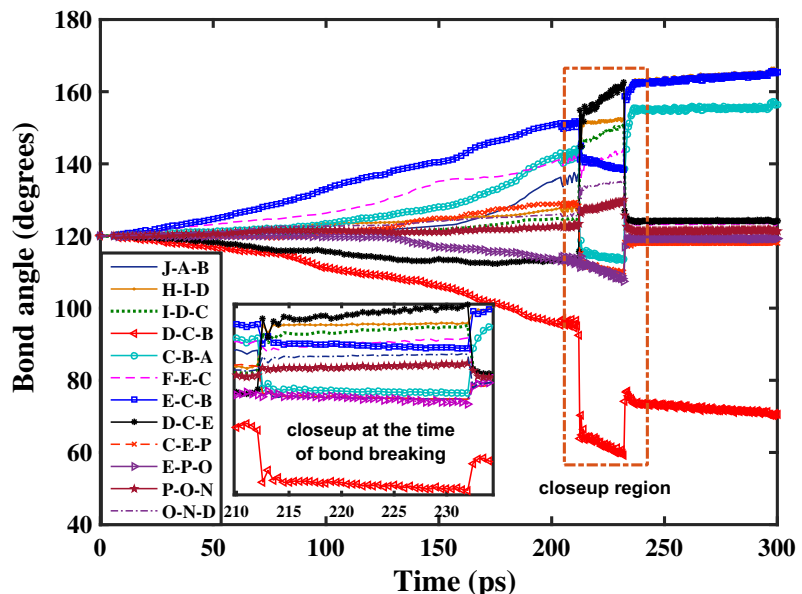

(c)

Fig. 5 a Atom position in the initial configuration when the lattice is oriented along the $13.9^{\circ}$, along with the atoms around the crack tip. Variation of the $\mathbf{b}$ bond length, $\mathbf{c}$ bond angles and $\mathbf{d}$

orientation is plotted in Fig. 4b, where the picture on the top shows a closeup of the selected region. It can be seen that the bond $\mathrm{B}-\mathrm{C}$ starts initially to elongate, to reach a bond length of $1.8 \AA$. Subsequently, the nearby bonds also start elongating, to reach the bond length of $\approx 1.8 \AA$. When all the bonds around the crack tip reach the critical bond length, the bond $\mathrm{C}-\mathrm{D}$ breaks first to create the first fracture. Results indicate that the bond length is the critical parameter to estimate fracture in Graphene. The corresponding change of bond angles with time are plotted in Fig. 4c. However, the bond rotation plotted in Fig. 4c cannot be considered as a parameter to estimate the bond breaking in Graphene. This is due to the following reasons: (i) oscillations in the bond angle until the first bond break; (ii) the bond

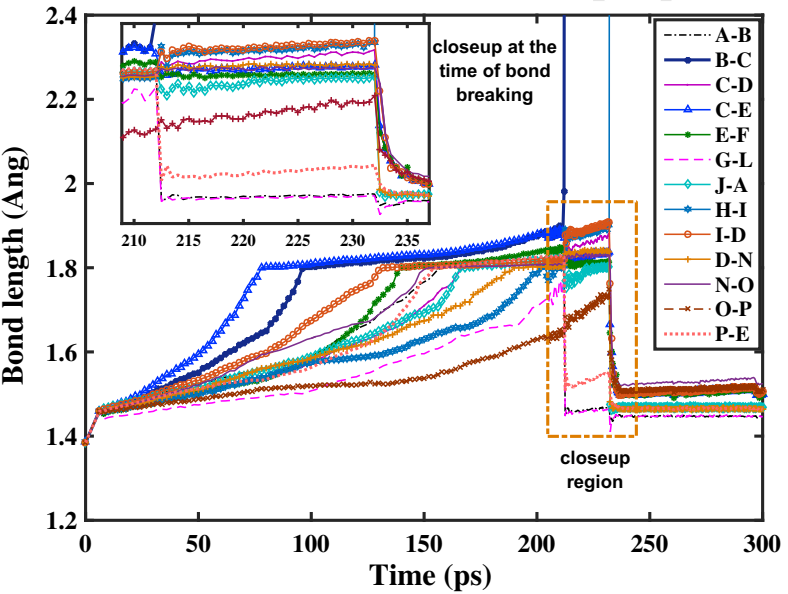

(b)

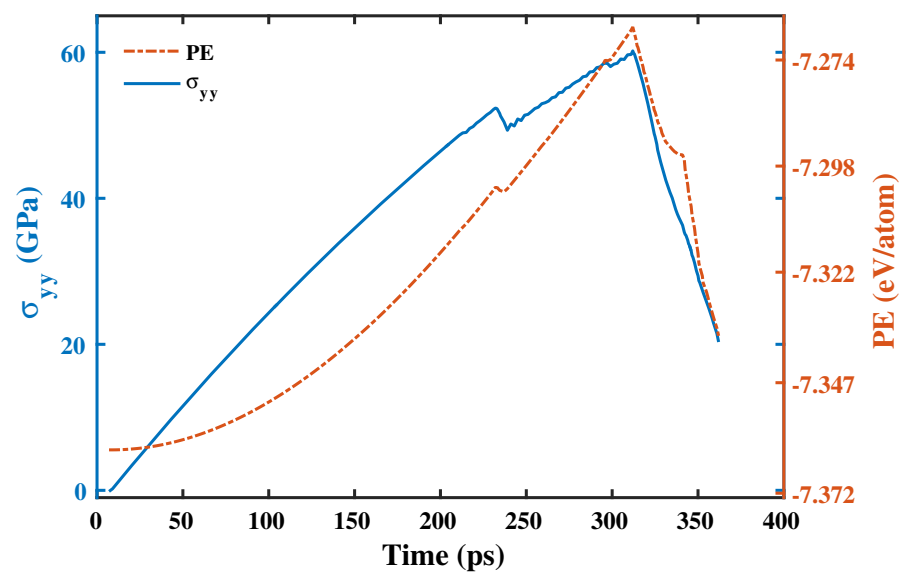

(d)

strain along the $y$ direction and the potential energy per atom, with time. The pictures in the bottom of $\mathbf{c}$ shows a closeup at the time of bond breaking. Plot $\mathbf{d}$ is generated for $\mathrm{a}_{0}=0.5 \mathrm{~L}$

angles of the bonds around the crack tip change significantly due to reorientation of the bonds after the first bond break. Therefore, bond length can be considered as a parameter to predict the bond break in Graphene.

A similar trend and mechanical behaviour in bond stretching is observed when the lattice is oriented along the $13.9^{\circ}$ and $30^{\circ}$, see Figs. $5 \mathrm{~b}$ and $6 \mathrm{~b}$, respectively. The bonds around the crack tip started to stretch first, to reach a critical value. When all the bonds around the crack tip reach the critical value, failure takes place. The bond angles also follow a similar trend, but they cannot be considered as a parameter to predict the fracture for the reasons explained above. Variation of the stress in the loading direction $\left(\sigma_{y y}\right)$ and the potential energy per 


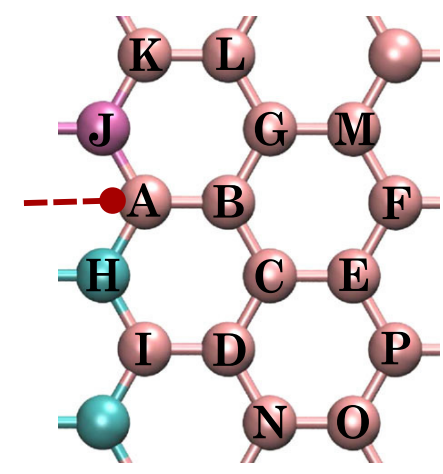

(a) $30^{\circ}$

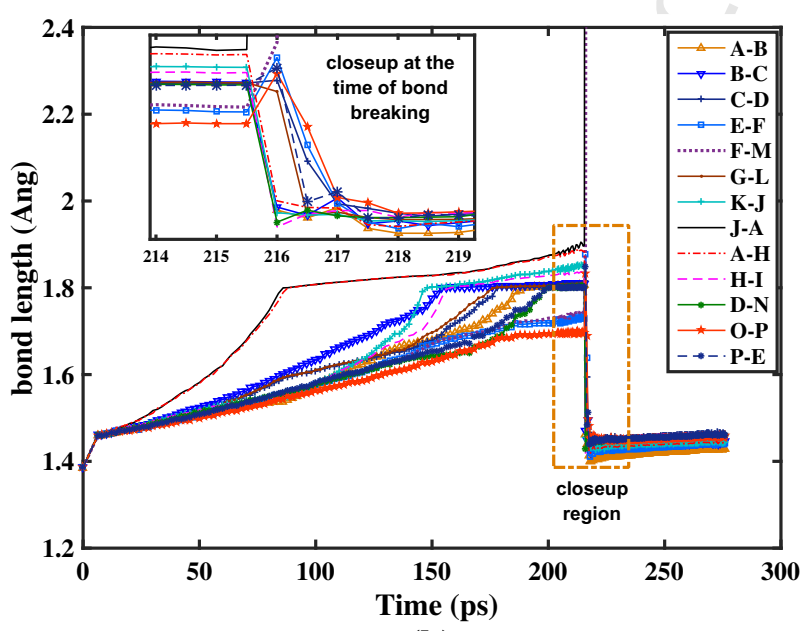

(b)

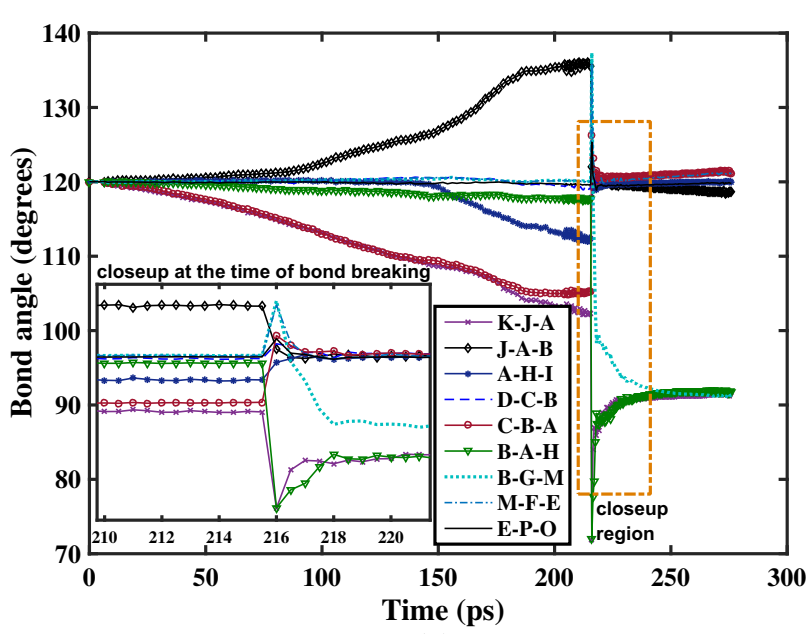

(c)

Fig. 6 a Atom position in the initial configuration when the lattice is oriented along the $30^{\circ}$, along with the atoms around the crack tip. Variation of the $\mathbf{b}$ bond length, $\mathbf{c}$ bond angles and $\mathbf{d}$

atom with time, when the lattice is oriented along $0^{\circ}$, $13.9^{\circ}$ and $30^{\circ}$, is plotted in Figs. $4 \mathrm{~d}, 5 \mathrm{~d}$ and $6 \mathrm{~d}$, respectively. The first drop in stress as well as potential energy is considered for the estimation of the yield stress and yield strain of the materials. Based on Fig. 5d, it is interesting to note that the $13.9^{\circ}$ lattice orientation shows a continuous increase in stress as well as potential energy even after the first bond break which is observed at 213.5 ps, refer to the closeup of Fig. 5b. The increase in stress as well as potential energy is continued till the second bond breaks at $232.5 \mathrm{ps}$. Therefore, results indicate that special orientations of Graphene can lead to improvement in the mechanical properties even after first bond failure.

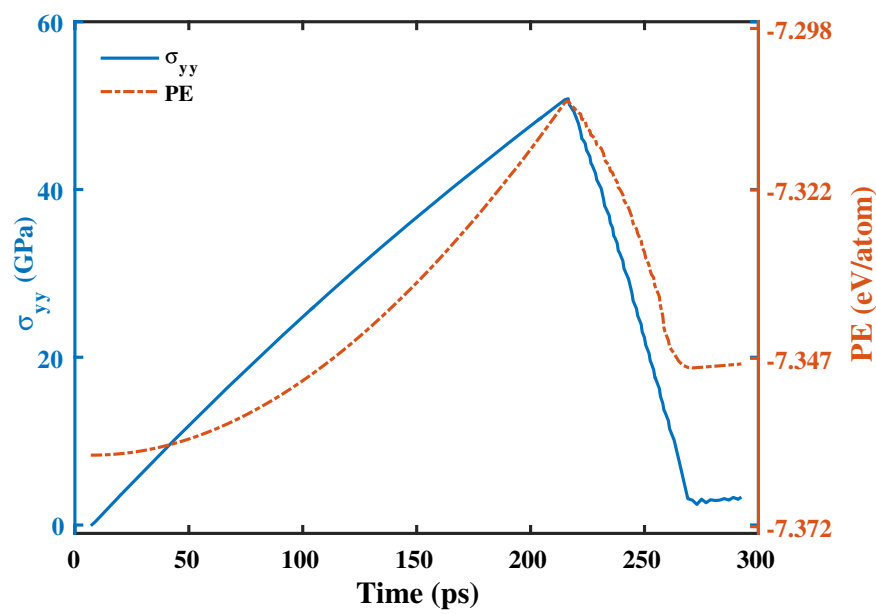

(d)

strain along the $y$ direction and the potential energy per atom, with time. The pictures in the bottom in (c) shows a closeup at the time of bond breaking. Plot $\mathbf{d}$ is generated for $\mathrm{a}_{0}=0.5 \mathrm{~L}$

\subsection{Lattice orientation dependent mechanical properties}

In order to understand the effect of lattice orientation on mechanical properties of Graphene, the tensile stressstrain curves have been generated for all the 10 different orientations considered in the present work. Variation of the stress with strain, for different lattice orientations is plotted in Fig. 7. Based on Fig. 7, it is observed that at zero strain all the orientations of Graphene have zero stress, which indicates that no residual stress exist in any of the configurations. Further strain controlled loading leads to continuous increase in strain which correspondingly leads to increase in stress. Due to the 
Fig. 7 Stress-strain plots of all the ten different configurations considered in the present work

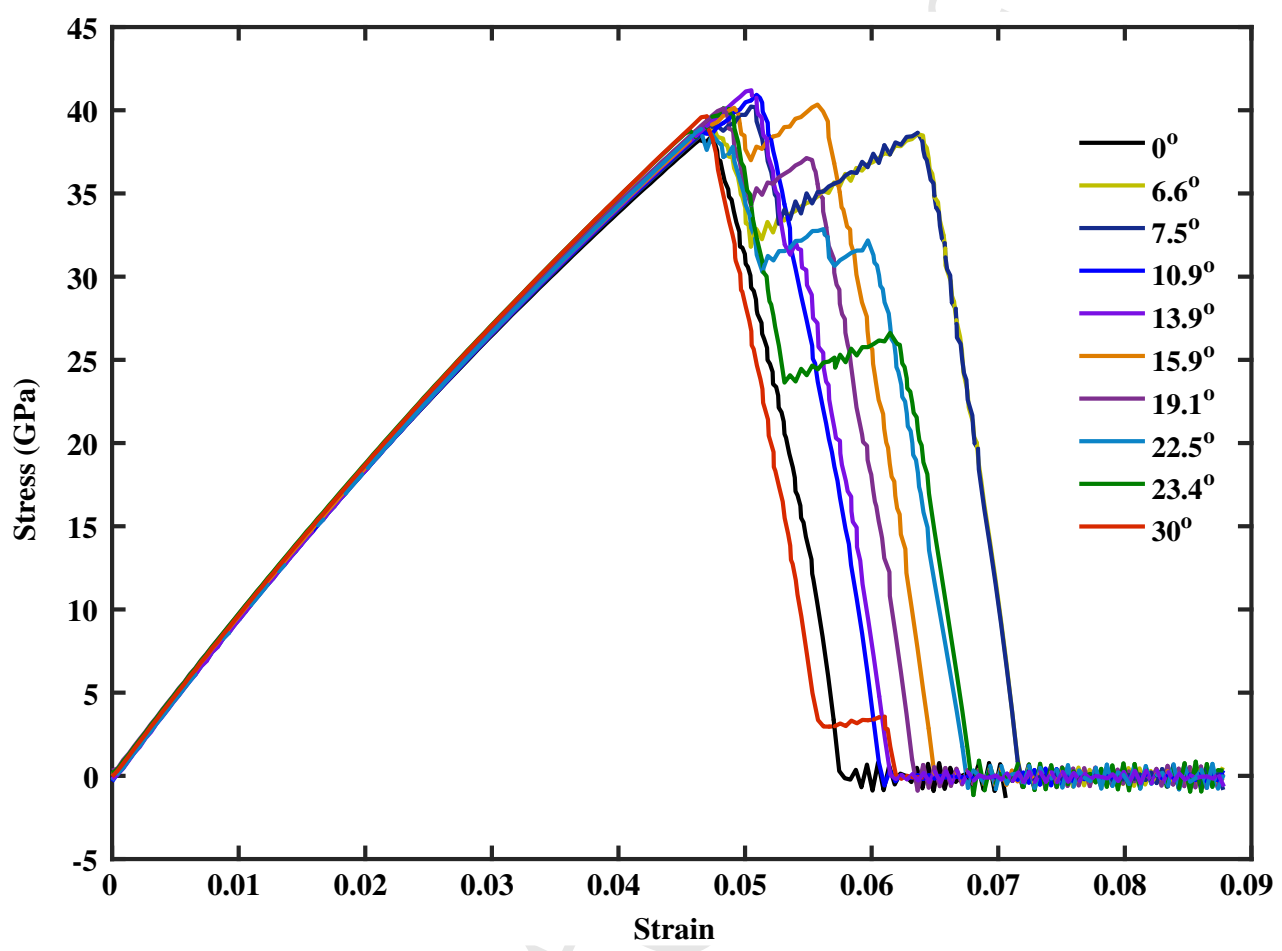

increase in strain, the bond length of an initial relaxed Graphene starts to increase. The atoms around the crack tip possess the highest potential energy. As soon as the bond lengths of the atoms around the crack tip reaches a critical value, the breaking of first bond occurs, indicated by a drop in the stress, leading to the yielding of Graphene. The corresponding stress and strain values are designated as yield stress and yield strain, respectively. Fluctuations in the yield properties of Graphene for different lattice orientations are observed. In order to study the effect of lattice orientation on yielding, yield stress and strain are plotted with lattice orientations in Fig. 8.

Figure $8 \mathrm{a}, \mathrm{b}$ shows the distribution of normalized yield stress and normalized yield strain with varying lattice orientations from $0^{\circ}$ (arm-chair) to $30^{\circ}$ (zig-zag), respectively. The normalization is based on the yield values at $0^{\circ}$. The arm-chair Graphene is observed to possess the lowest yield stress, which further increases with increase in lattice orientation angle and reaches a maximum at $13.9^{\circ}$. Subsequent increase in lattice orientation leads to the reduction in yield stress until $22.5^{\circ}$. The yield stress is observed to increase with the lattice orientation until $30^{\circ}$. From Fig. 8a, the zig-zag configuration is observed to possess a higher yield stress as compared to the armchair Graphene. Also a similar trend in yield strain is observed. From Fig. 8b, it can be noticed that the armchair Graphene has a lower yield strain as compared to the zig-zag configuration. Yield strain also shows an increase in value by increasing lattice orientation and reaches maximum at $13.9^{\circ}$. Subsequent increase in lattice orientation leads to a drop in yield strain until $23.4^{\circ}$. Later on, the yield strain is observed to increase until $30^{\circ}$.

The state of equilibrium energy of an atomistic system depends on the arrangement of atoms in that particular configuration. The structure will be stable when the system potential energy is minimum. In Graphene, the bonds along the loading direction will undergo more deformation and are responsible for failure with very similar bond elongation at the fracture point (Zhao et al. 2009). Furthermore, the magnitude of the bond length and bond angle variation in the zig-zag direction is reported to be much larger than that of the arm-chair direction (Zhao et al. 2009). This indicates that the zig-zag configuration is energetically more stable and absorbs more energy before fracture, as compared to the arm-chair configuration. In other words, the arrangement of atoms in the arm-chair Graphene leads to an energetically unstable configuration. In this work, we observed that the stability increases with lattice orientation reaching the maximum at $13.9^{\circ}$. Therefore, the potential energy is observed to be the lowest when the 


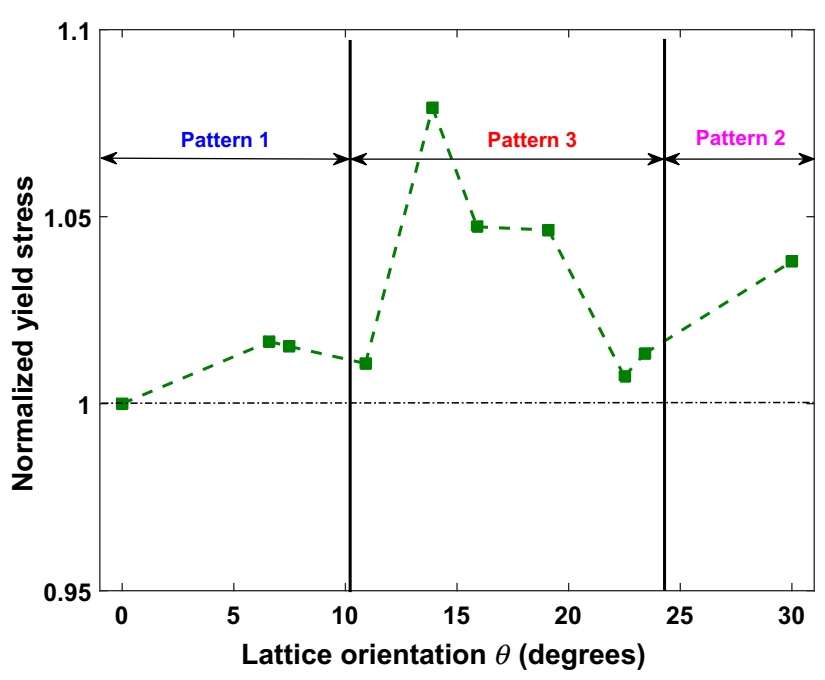

(a)

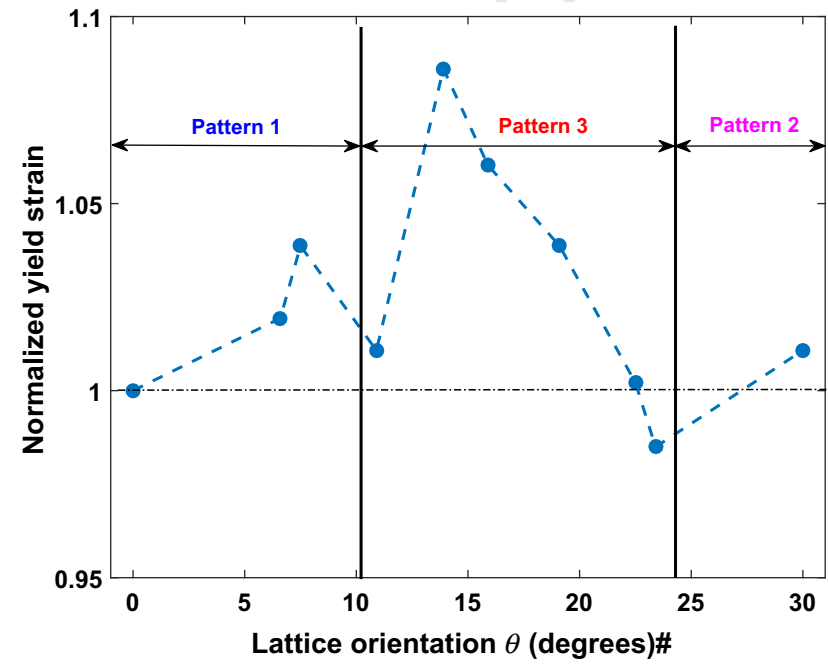

(b)

Fig. 8 Distribution of the a normalized stress and $\mathbf{b}$ normalized strain with the lattice orientation. The plots are generated based on the results with $a_{0}=0.5 \mathrm{~L}$

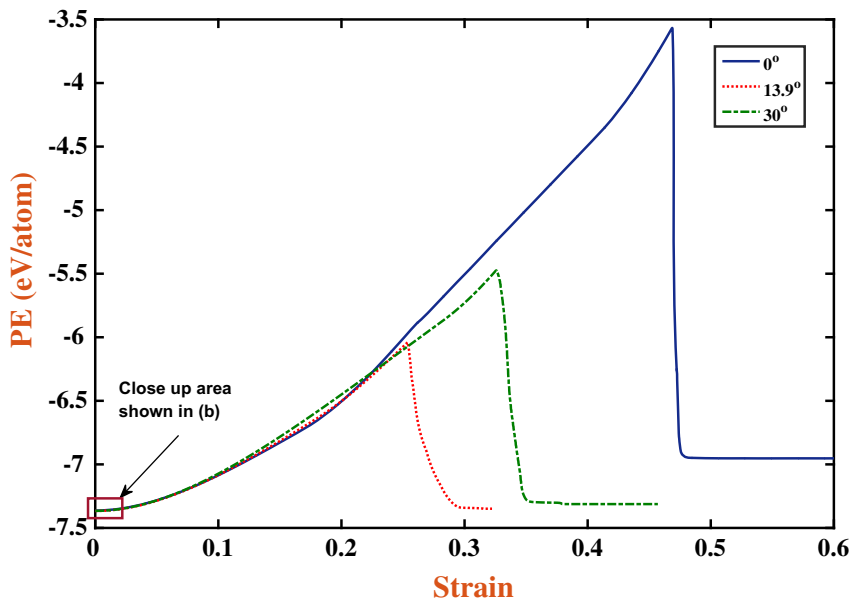

(a)

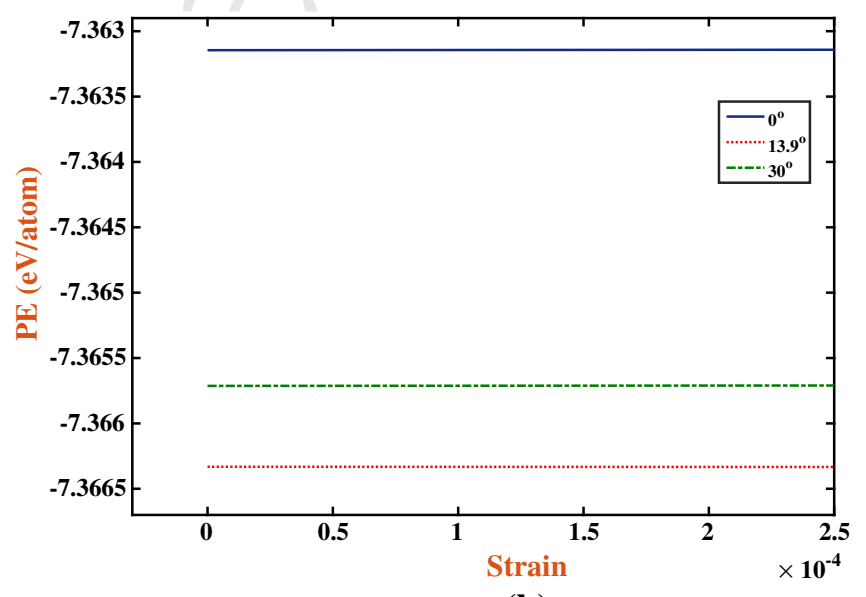

(b)

Fig. 9 Distribution of the a potential energy with strain and $\mathbf{b}$ a close up at the beginning, with zero strain. The plots are generated based on the initial potential energy of the system without any crack, after the minimization

lattice is oriented along $13.9^{\circ}$, as shown in Fig. 9. This explains the reasons for the highest potential energy of the arm-chair Graphene.

The lower fracture strength of arm-chair $\left(0^{\circ}\right)$ Graphene as compared to the zig-zag $\left(30^{\circ}\right)$ Graphene can be correlated to the system potential energy plotted in Fig. 9. Based on Fig. 9b, the arm-chair Graphene possesses the highest initial potential energy of $-7.3631 \mathrm{eV} /$ atom, as compared to other configurations considered in the present study. Due to the highest initial potential energy of the arm-chair Graphene, an early yielding is anticipated. Whereas, the zig-zag Graphene is observed to possess an initial potential energy of $-7.3657 \mathrm{eV} /$ atom, which is lower than the arm-chair Graphene. This indicates that the zig-zag configuration is more stable and hence it can sustain more strain, which leads to a higher fracture strength as compared to the arm-chair Graphene. Interestingly, the Graphene with chiral vector $(2,5)$ at $13.9^{\circ}$ lattice orientation is found to be the most stable configuration with an initial potential energy of $-7.3663 \mathrm{eV} / \mathrm{atom}$, out of the 10 orientations considered in the present study. This is further confirmed by the highest fracture strength of Graphene when the lattice is oriented at $13.9^{\circ}$, refer to Fig. 8a. 
Interestingly, the crack patterns discussed in Sect. 3.1 for different lattice orientations can be correlated to the yield stress and yield strain values. The pattern number 1 in Fig. $2 \mathrm{~b}$ is observed in $0^{\circ}$ (arm-chair), $6.6^{\circ}$, and $10.9^{\circ}$ configurations. However, pattern number 2 is observed only in $30^{\circ}$ (zig-zag) orientation. The remaining lattice orientations $\left(7.5^{\circ}, 13.9^{\circ}, 15.9^{\circ}, 19.1^{\circ}, 22.5^{\circ}\right.$, and $23.4^{\circ}$ ) are exhibiting the pattern number 3 . In the first pattern, the crack initiation and growth takes place perpendicular to the loading direction, which corresponds to the arm-chair $\left(0^{\circ}\right)$ configuration. Similar crack initiation and growth perpendicular to the loading direction is observed in the third pattern as well, which corresponds to the zig-zag $\left(30^{\circ}\right)$ configuration. Therefore, the crack orientations between $0^{\circ}$ to $10.9^{\circ}$ are mainly influenced by the arm-chair pattern. On the other hand, crack orientations between $22.5^{\circ}$ to $30^{\circ}$ are mainly influenced by the zig-zag pattern. The other orientations are influenced by the pattern number 3. Results demonstrate that the pattern number 1 is the weakest and the pattern number 2 is the strongest. Based on the results, $\approx 10 \%$ improvement as compared to an initial arm-chair configuration in yield stress as well as yield strain could be achieved by selecting the lattice orientation in relation to the initial notch direction.

\subsection{Crack size dependent mechanical properties}

The variation of stress with strain for arm-chair Graphene at different percentage of crack length with respect to the width of the sample is analyzed at $0 \mathrm{~K}$, see Fig. 10a. The crack size is varied from $\mathrm{a}_{0}=0.025 \mathrm{~L}$ to $0.90 \mathrm{~L}$ with total 13 different initial simulated crack lengths. With the increase in crack size, the amount of energy required to break the bond around the crack tip decreases. Hence, the yield stress is also observed to be decreasing, as shown in Fig. 10a. It is observed that until $\mathrm{a}_{0}=0.10 \mathrm{~L}$ of the crack size, the stiffness remains almost the same. The trend observed in Fig. 10a is observed to be similar to the variation of stress with strain for different hole sizes in Zhang et al. (2012a). However, further increase in crack size leads to decrease in yield stress as well as the stiffness. On the other hand, the yield strain is noticed to decrease initially with an increase in crack size. Whereas, after a critical crack size, $a_{0} \approx 0.20 \mathrm{~L}$, the variation in the yield strain remains almost constant at 0.05 . Also sim- ilar variations are observed for the zig-zag Graphene at $\approx 0 \mathrm{~K}$, as shown in Fig. 10c.

In order to understand the effect of temperature on the mechanical properties of Graphene, preliminary simulations are performed at room temperature $300 \mathrm{~K}$, for arm-chair and zig-zag orientations with varying crack length, as shown in Fig. 10b, d, respectively. It is observed that even at $300 \mathrm{~K}$, the yield strain becomes constant after a critical crack length. The variation of the fracture toughness with $\mathrm{a}_{0} / \mathrm{L}$ ratio for arm-chair and zig-zag orientations at $\approx 0$ and $300 \mathrm{~K}$ are plotted in Fig. 10e. From these results, both the arm-chair as well as the zig-zag orientations show almost constant fracture toughness values at different temperatures. In general, $\mathrm{a}_{0} / \mathrm{L}<0.5$ can be considered to predict the stress-intensity factor. From Fig. 10e, the median values of mode I fracture toughness of arm-chair/zig-zag Graphene is found to be $8.4 \mathrm{MPa} \sqrt{m}$ at $\approx 0 \mathrm{~K}$. The variation in standard deviation is found to be 1.27 . On the other hand, at $300 \mathrm{~K}$, the median values of mode I fracture toughness of arm-chair/zig-zag Graphene is found to be $7.4 \mathrm{MPa} \sqrt{m}$. The variation in standard deviation is found to be 1.52. A further detailed study on the stress intensity factor of single layer Graphene at different temperatures is beyond the scope of present work and is left for future investigation.

Furthermore, to study the combined effect of different orientations and crack sizes on the yield stress and yield strain, 10 different lattice orientations with 13 different crack sizes are simulated. The distribution of yield stress and yield strain, with crack length is plotted in Fig. 11a, b, respectively. Figure 11c shows the stress-strain distribution with lattice lattice orientation in a three dimensional plot, for all the initial crack lengths considered in this paper. Based on Fig. 10a, for a given orientation, the yield stress decreases with an increase in crack length, as discussed in Sect. 3.2. Note that the discussion in Sect. 3.2 is valid for crack sizes of $\mathrm{a}_{0} \geq 0.20 \mathrm{~L}$. Smaller crack sizes shows different variation in yield stress with varying crack orientations due to the involvement of the free surface near the crack tip. The yield strain also shows a decreasing trend with increase in crack size for a given orientation up to $\mathrm{a}_{0}=0.33 \mathrm{~L}$ of crack length. A constant yield strain of $\approx 0.05$ is observed with further increase in crack size up to $\mathrm{a}_{0}=0.90 \mathrm{~L}$. We report that this is one of the reasons for the softening of the Graphene, as displayed by the decrease of stiffness with increase in crack length. 


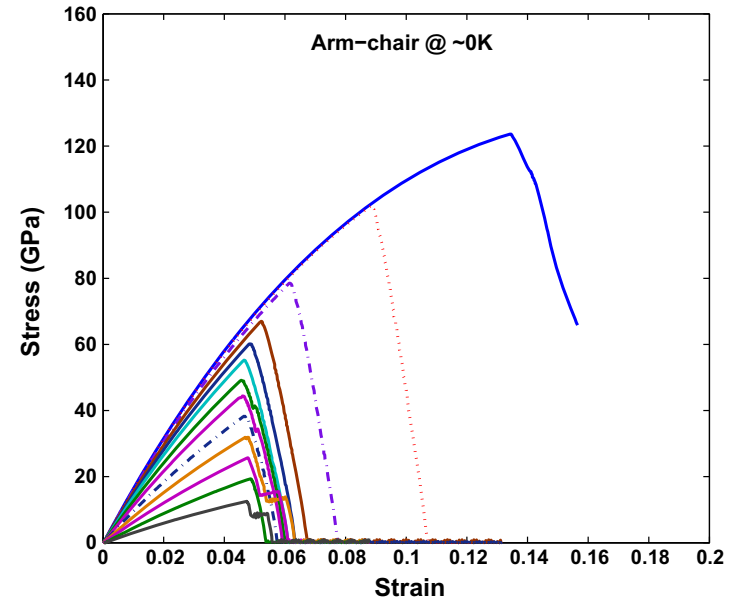

(a)

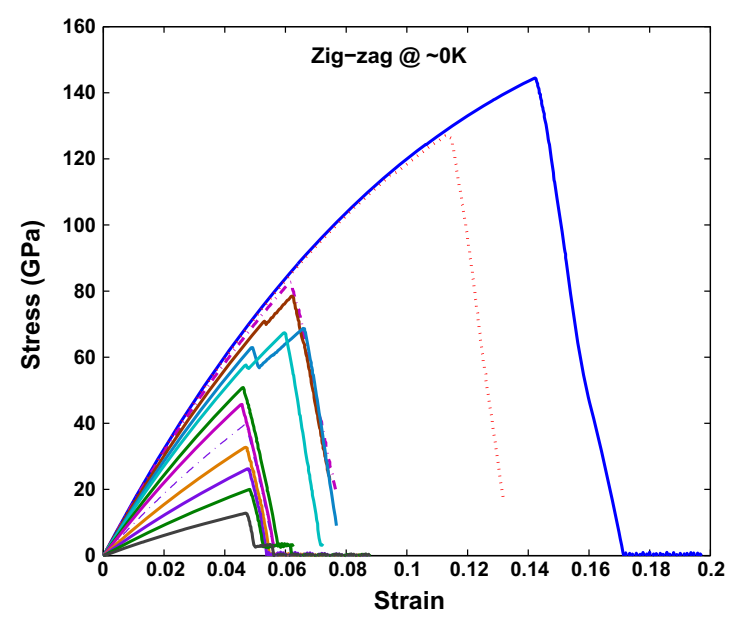

(c)

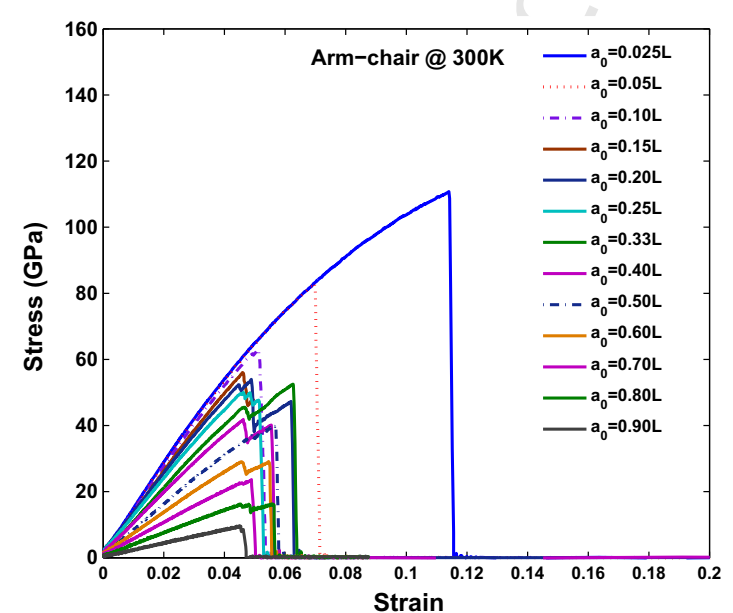

(b)

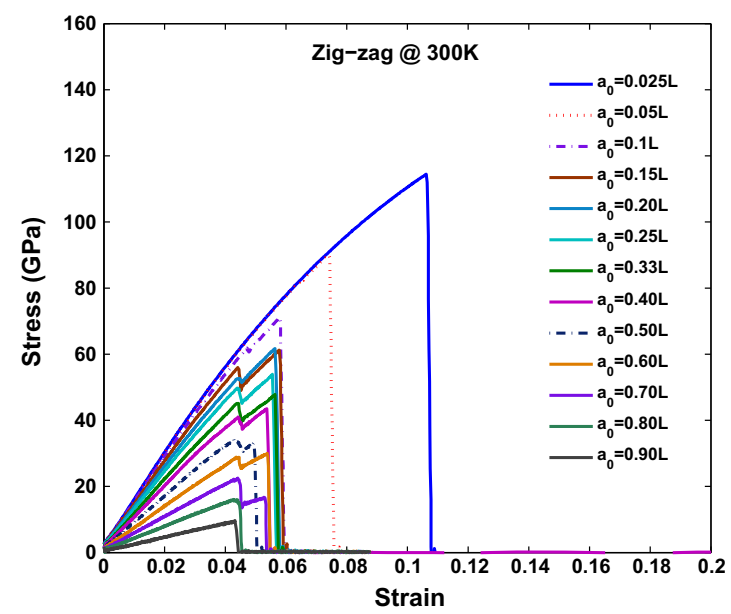

(d)

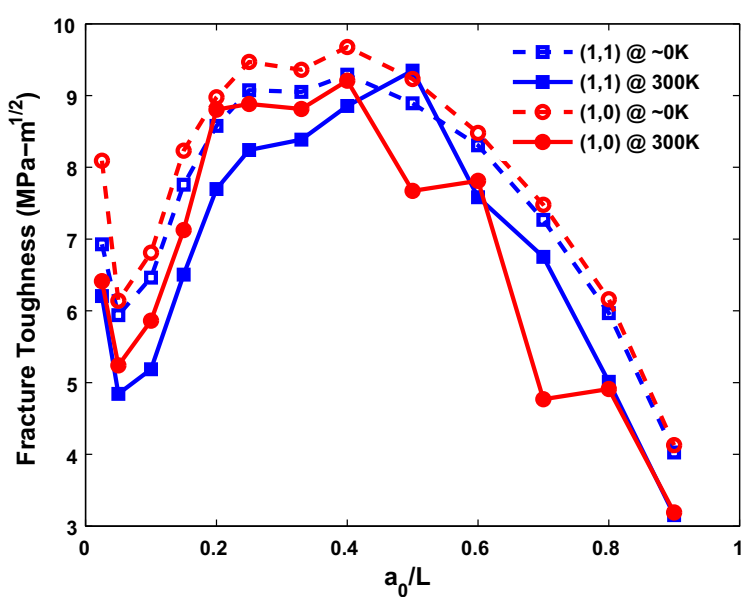

(e)

Fig. 10 Stress-strain plots for $\mathbf{a}, \mathbf{b}$ arm-chair and $\mathbf{c}, \mathbf{d}$ zig-zag Graphene at $\approx 0$ and $300 \mathrm{~K}$, respectively, for different crack lengths. e Variation of fracture toughness in arm-chair and zig-zag Graphene at $\approx 0$ and $300 \mathrm{~K}$ 


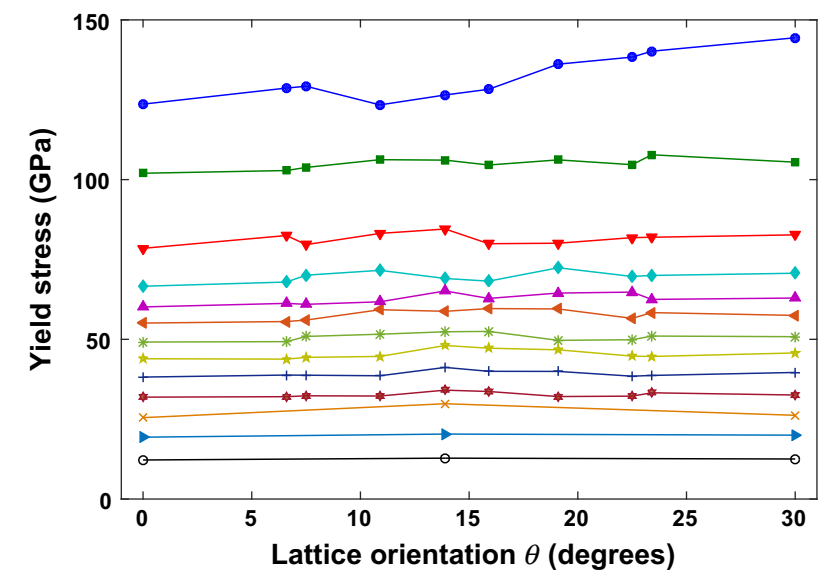

(a)

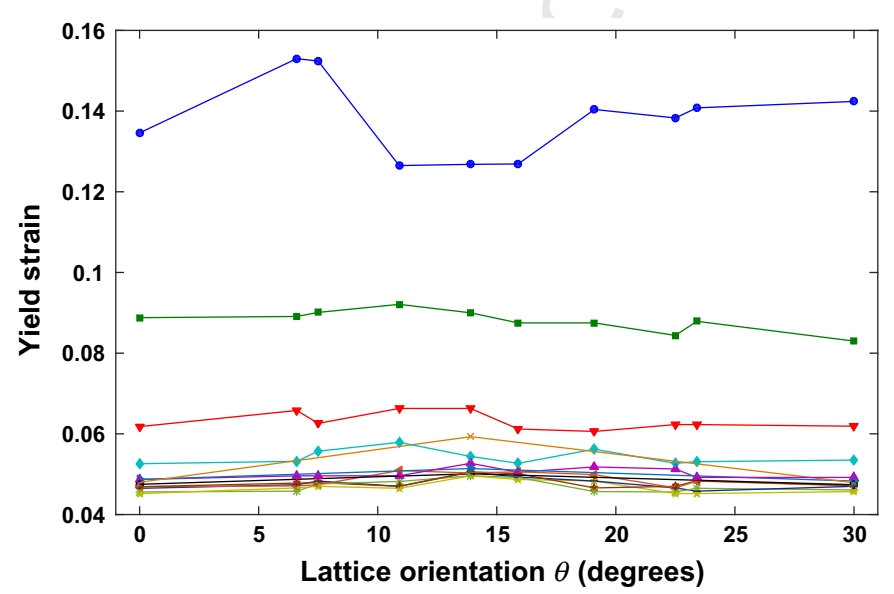

(b)

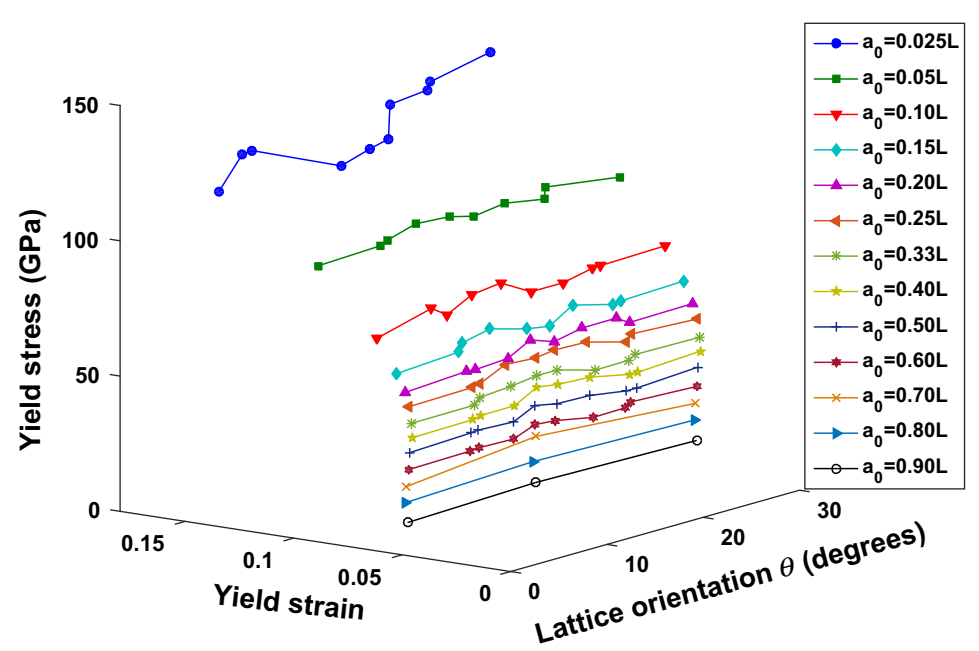

(c)

Fig. 11 Variation of the a stress and $\mathbf{b}$ strain, with the lattice orientation, for various crack sizes. $\mathbf{c}$ Plots $\mathbf{a}$ and $\mathbf{b}$ are combined to generate the three dimensional plot, where the variation of

Furthermore, based on the yield stress and yield strain plotted in Fig. 11a, b, a sudden decrease in yield stress and yield strain can be noticed for crack sizes $<10 \mathrm{~nm}$. However, for larger crack sizes a linear decrease in yield stress is observed, whereas yield strain remains constant. The behaviour is found to be the same in all the lattice orientations considered in the present study. Therefore, the behaviour of yield stress as well as yield strain with crack length is insensitive to the orientation, as shown in Fig. 12a, b. The variation of the yield stress with yield strain for all the orientations considered in the present work is plotted Fig. 12c. Based on the results, a yield strain of $\approx 0.05$ can be considered as a critical strain value below which Graphene the yield stress and yield strain with the crack orientation can be simultaneously monitored. The legends of plots $\mathbf{a}$ and $\mathbf{b}$ are shown in (c)

does not show failure. This information can be utilized in the design of nano-devices for various strain sensor applications.

\section{Conclusions}

Tensile deformation of two dimensional Graphene structure with an edge crack has been simulated based on molecular dynamics. Application of the load leads to the bond deformation, resulting in an increase of the system potential energy and hence the stress along the loading direction. The severely stressed bonds are breaking when the stress reaches a critical value, 


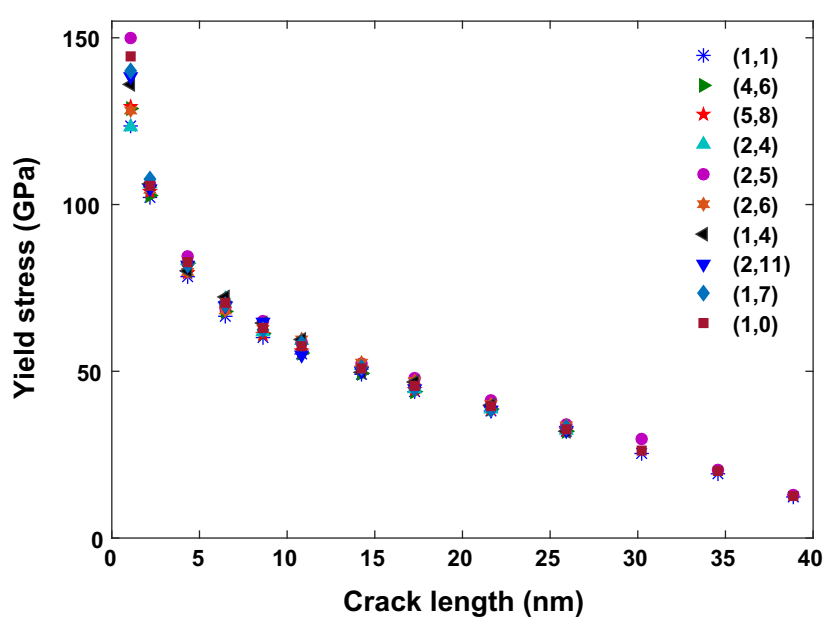

(a)

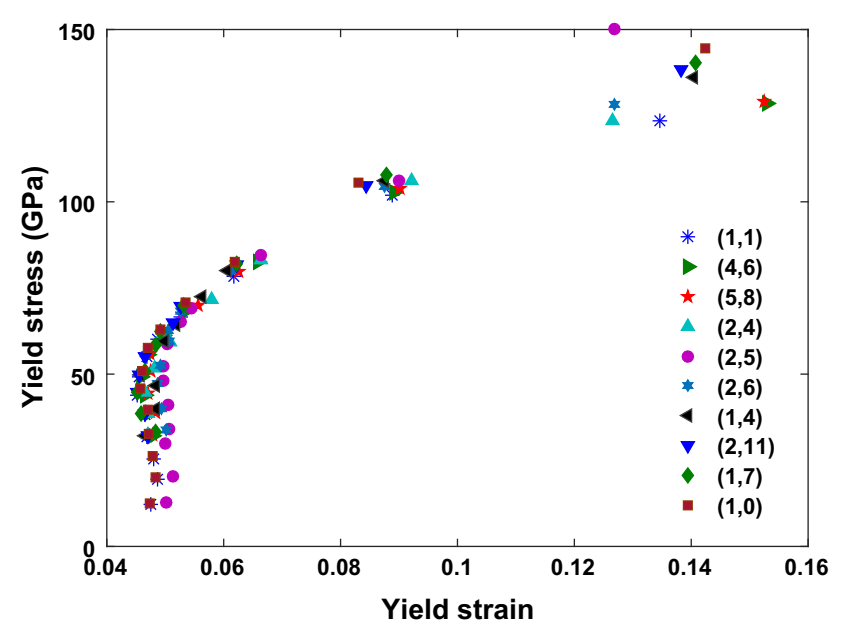

(c)

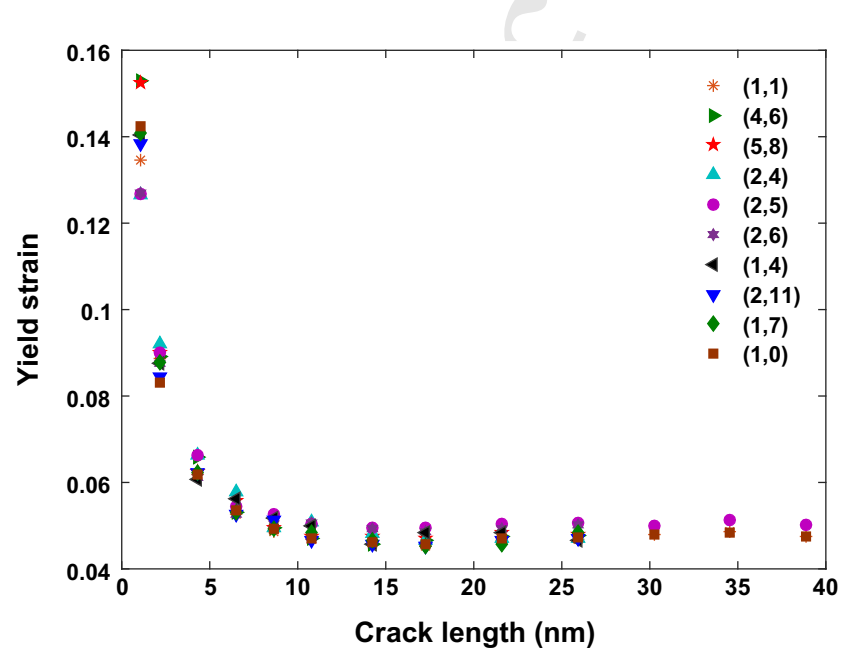

(b) exposing the new set of atoms to resist the applied load.

Ten different lattice orientations with thirteen different initial crack lengths have been considered to study their effect on the yield stress and yield strain of Graphene. Graphene is observed fracture in three particular patterns in all the lattice orientations, where the arm-chair fracture pattern is observed to possess the lowest yield properties. A sudden decrease in yield stress and yield strain is noticed for crack sizes $<10 \mathrm{~nm}$. However, for larger crack sizes a linear decrease in yield stress is noticed, whereas a constant yield strain of $\approx 0.05$ is observed. Therefore, the yield strain of $\approx 0.05$ can be considered as a critical strain value below which Graphene does not show failure. This information can be utilized in the design of nano-devices for various strain sensor applications. Mode I fracture toughness of arm-chair and zig-zag Graphene is estimated as $8.4 \pm 1.27 \mathrm{MPa} \sqrt{m}$ and $7.4 \pm 1.52 \mathrm{MPa} \sqrt{m}$ at $\approx 0$ and $300 \mathrm{~K}$, respectively. Furthermore, Graphene can be coated on the Silicon surface to enhance the mechanical (Berardone et al. 2014; Schröder et al. 2012) and electrical characteristics the solar cells (Köntges et al. 2011; Paggi et al. 2011, 2013, 2014). The present study will be useful in selecting the optimum orientation of Graphene.

Acknowledgments B. Javvaji, D. R. Mahaptra and T. Rabczuk gratefully acknowledge the financial support from the Germany Science Foundation (DFG) and from the International Research Staff Exchange Scheme (IRSES), FP7-PEOPLE-2010-IRSES, through the project 'MultiFrac'. M. Paggi and P. R. Budarapu acknowledges the funding from the European Research Coun- 
cil (ERC), Grant No. 306622 through the ERC Starting Grant "Multi-field and multi-scale Computational Approach to Design and Durability of PhotoVoltaic Modules"-CA2PVM. B. Javvaji and D. R. Mahapatra thankfully acknowledge the use of computational facilities at the ACECOST Computational Science Lab, Department of Aerospace Engineering, IISc and funding under ACECOST Phase-III program of Aeronautics Research and Development Board, India. Zi appreciates the financial support through Grant No. 20133010021770, from the New \& Renewable Energy Core Technology Program of the Korea Institute of Energy Technology Evaluation and Planning (KETEP), Ministry of Trade, Industry \& Energy, Republic of Korea.

\section{Appendix: Tersoff potential function}

The mathematical expression of the bond energy based on the Tersoff potential is given in Eq. (1). The bond energy in the Tersoff framework is a combination of repulsive $\left(f_{R}\right)$ energy function which is exponentially decaying and attractive $\left(f_{A}\right)$ energy function that exponentially increases; with the increase of distance between the atoms. $f_{c}$ is a smooth spherical cutoff function around atom $\alpha$ based upon the distance to the first nearest-neighbor shell. The function $f_{c}$ in Eq. (1) is defined as Tersoff (1989):

$$
\begin{aligned}
& f_{c}\left(r_{\alpha \beta}\right) \\
& \quad= \begin{cases}1 & \text { when } r_{\alpha \beta}<\mathcal{R}_{\alpha \beta} \\
\frac{1}{2}+\frac{1}{2} \cos \left(\frac{\pi\left(r_{\alpha \beta}-\mathcal{R}_{\alpha \beta}\right)}{\left(\mathcal{S}_{\alpha \beta}-\mathcal{R}_{\alpha \beta}\right)}\right) & \text { when } \mathcal{R}_{\alpha \beta}<r_{\alpha \beta}<\mathcal{S}_{\alpha \beta} \\
0 & \text { when } r_{\alpha \beta}>\mathcal{S}_{\alpha \beta}\end{cases}
\end{aligned}
$$

from Eq. (3), $f_{c}$ returns a value of 1 if $r_{\alpha \beta}$ is less than $\mathcal{R}_{\alpha \beta}$ and 0 when $r_{\alpha \beta}$ greater than $\mathcal{S}_{\alpha \beta}$. The values of the constants $\mathcal{R}_{\alpha \beta}=\sqrt{\mathcal{R}_{\alpha} \mathcal{R}_{\beta}}$ and $\mathcal{S}_{\alpha \beta}=\sqrt{\mathcal{S}_{\alpha} \mathcal{S}_{\beta}}$, (where $\alpha$ and $\beta$ can be two different atom types, like Silicon and Carbon) are listed for Silicon and Carbon atoms in Tersoff (1989). The repulsive and attractive potential energies are tuned with the parameters $\mathcal{A}_{\alpha \beta}$ and $\mathcal{B}_{\alpha \beta}$, respectively. The repulsive potential energy is defined as Tersoff (1989)

$f_{R}\left(r_{\alpha \beta}\right)=\mathcal{A}_{\alpha \beta} e^{-\mathcal{D}_{\alpha \beta} r_{\alpha \beta}}$

and the attractive potential energy is estimated from Tersoff (1989)

$f_{A}\left(r_{\alpha \beta}\right)=-\mathcal{B}_{\alpha \beta} e^{-\mathcal{E}_{\alpha \beta} r_{\alpha \beta}}$

where $\mathcal{A}=\sqrt{\mathcal{A}_{\alpha} \mathcal{A}_{\beta}}, \mathcal{B}=\sqrt{\mathcal{B}_{\alpha} \mathcal{B}_{\beta}}, \mathcal{D}_{\alpha \beta}=\left(\mathcal{D}_{\alpha}+\right.$ $\left.\mathcal{D}_{\beta}\right) / 2$ and $\mathcal{E}_{\alpha \beta}=\left(\mathcal{E}_{\alpha}+\mathcal{E}_{\beta}\right) / 2$, in Eqs. (4) and (5) are constants. The variable $b_{\alpha \beta}$ in Eq. (1) is designed to represent the bond strength of the potential. $b_{\alpha \beta}$ is inversely proportional to the coordination number and is defined as Tersoff (1989)

$b_{\alpha \beta}=\xi_{\alpha \beta}\left(1+\mathcal{P}_{\alpha}^{q_{\alpha}} \zeta_{\alpha \beta}^{q_{\alpha}}\right)^{-1 / 2 q_{\alpha}}$

where $\mathcal{P}$ and $q$ are the constants. $\zeta_{\alpha \beta}$ provides a weighted measure of the number of other bonds $(\gamma)$ competing with the bond $\alpha-\beta$, which is defined as Tersoff (1989)

$$
\zeta_{\alpha \beta}=\sum_{\gamma \neq \alpha, \beta} f_{c}\left(r_{\alpha \gamma}\right) g\left(\theta_{\alpha \beta} \gamma\right)
$$

where $\xi_{\alpha \beta}$ is the strengthening or weakening factor of the hetero-polar bonds and $g\left(\theta_{\alpha} \beta \gamma\right)$ provides a measure of dependence on the bonding angle $\theta_{\alpha \beta \gamma}$, subtended at atom $\alpha$ by atoms $\beta$ and $\gamma$. The variable $g\left(\theta_{\alpha} \beta \gamma\right)$ is included to stabilize the atomic geometry under shear operations and to provide an effective coordination contribution based on the elastic energy of the current configuration, which is defined as Tersoff (1989)

$g\left(\theta_{\alpha \beta \gamma}\right)=1+\frac{c_{\alpha}^{2}}{d_{\alpha}^{2}}-\frac{c_{\alpha}^{2}}{d_{\alpha}^{2}+\left(h_{\alpha}-\cos \left(\theta_{\alpha \beta \gamma}\right)\right)^{2}}$

where $c_{\alpha}, d_{\alpha}$ and $h_{\alpha}$ are the constants.

720 721

\section{References}

Ansari R, Ajori S, Motevalli B (2012) Mechanical properties of defective single-layered graphene sheets via molecular dynamics simulation. Superlattices Microstruct 51:274289

Berardone I, Corrado M, Paggi M (2014) A generalized electric model for mono and polycrystalline silicon in the presence of cracks and random defects. Energy Procedia 55:22-29

Bu H, Chen Y, Zou M, Yi H, Bi K, Ni Z (2009) Atomistic simulations of mechanical properties of graphene nanoribbons. Phys Lett A 373:3359-3362

Budarapu P, Javvaji B, Sutrakar V, Mahapatra D, Zi G, Rabczuk $\mathrm{T}$ (2015) Crack propagation in graphene. J Appl Phys 118:382-395

Budarapu P, Rammohan B, Vijay S, Satish B, Raghunathan R (2009) Aero-elastic analysis of stiffened composite wing structure. J Vib Eng Technol 8(3):255-264

Budarapu P, Sudhir S, Brahmanandam J, Mahapatra D (2014c) Vibration analysis of multi-walled carbon nanotubes embedded in elastic medium. Front Struct Civil Eng $8(2): 151-159$

Cao G (2014) Atomistic studies of mechanical properties of graphene. Polymers 6:2404-2432

Changgu L, Xiaoding W, Jeffrey WK, James H (2008) Measurement of the elastic properties and intrinsic strength of monolayer graphene. Science 321(5887):385-388 
Chen K, Bin S, Yonghai Y, Juanjuan Q, Lin G (2015a) Binary synergy strengthening and toughening of bio-inspired nacrelike graphene oxide/sodium alginate composite paper. ACS Nano 9(8):8165-8175

Chen M, Quek S, Sha Z, Chiu C, Pei Q, Zhang Y (2015b) Effects of grain size, temperature and strain rate on the mechanical properties of polycrystalline graphene -a molecular dynamics study. Carbon 85:135-146

Chuvilin A, Meyer J, Algara-Siller G, Kaiser U (2009) From graphene constrictions to single carbon chains. New J Phys 11:083019

Datta D, Nadimpalli S, Yinfeng L, Shenoy V (2015) Effect of crack length and orientation on the mixed-mode fracture behavior of graphene. Extreme Mech Lett 5:10-17

Fengnian X, Han W, Di X, Madan D, Ashwin R (2014) Twodimensional material nanophotonics. Nat Photonics 8:899907

Fiori G, Bonaccorso F, Iannaccone G, Palacios T, Neumaier D, Seabaugh A, Banerjee S, Colombo L (2014) Electronics based on two-dimensional materials. Nat Nanotechnol 9:768-779

Gamboa A, Vignoles G, Leyssale J-M (2015) On the prediction of graphene's elastic properties with reactive empirical bond order potentials. Carbon 89:176-187

Geim A (2009) Graphene: status and prospects. Science 324:1530-1534

Grantab R, Shenoy V, Ruoff R (2010) Anomalous strength characteristics of tilt grain boundaries in graphene. Science 330:946-948

Hadden C, Klimek-McDonald D, Pineda E, King J, Reichanadter A, Miskioglu I, Gowtham S, Odegard G (2015) Mechanical properties of graphene nanoplatelet/carbon fiber/epoxy hybrid composites: Multiscale modeling and experiments. Carbon 95:100-112

Huhu C, Chuangang H, Yang Z, Liangti Q (2014) Graphene fiber: a new material platform for unique applications. NPG Asia Mater 6:e113

Jhon Y, Jhom Y, Yoem G, Jhon M (2014) Orientation dependence of the fracture behavior of graphene. Carbon 66:619-628

Jhon Y, Zhu S, Ahn J, Jhon M (2012) The mechanical responses of tilted and non-tilted grain boundaries in graphene. Carbon 50:3708-3716

Jin C, Lan H, Peng L, Suenaga K, Iijima S (2009) Deriving carbon atomic chains from graphene. Phys Rev Lett 102:205501

Khare R, Mielke S, Paci J, Zhang S, Ballarini R, Schatz G, Belytschko T (2007) Coupled quantum mechani$\mathrm{cal} /$ molecular mechanical modeling of the fracture of defective carbon nanotubes and graphene sheets. Phys Rev B 75:075412

Kim K, Artyukhov V, Regan W, Liu Y, Crommie M, Yakobson B, Zettl A (2012) Ripping graphene: preferred directions. Nano Lett 12:293-297

Kim Y, Lee J, Yeom M, Shin J, Kim H, Cui Y, Kysar J, Hone J, Jung Y, Jeon S, Han S (2013) Strengthening effect of single-atomic-layer graphene in metal-graphene nanolayered composites. Nat Commun 4:2114

Kinam K, Jae-Young C, Taek K, Seong-Ho C, Hyun-Jong C (2011) A role for graphene in silicon-based semiconductor devices. Nature 479:338-344
Köntges M, Kunze I, Kajari-Schröder S, Breitenmoser X, Bjrneklett B (2011) The risk of power loss in crystalline silicon based photovoltaic modules due to microcracks. Sol Energy Mater Sol Cells 95:1131-1137

Kostarelos K, Novoselov K (2014) Graphene devices for life. Nat Nanotechnol 9:744-745

Kravets V, Jalil R, Kim Y-J, Ansell D, Aznakayeva D, Thackray $\mathrm{B}$, Britnell L, Belle $\mathrm{B}$, Withers $\mathrm{F}$, Radko I, Han Z, Bozhevolnyi S, Novoselov K, Geim A, Grigorenko A (2014) Graphene-protected copper and silver plasmonics. Sci Rep 4:5517

Liu F, Ming P, Li J (2007) Ab initio calculation of ideal strength and phonon instability of graphene under tension. Phys Rev B 76:064120

Liu J (2014) Charging graphene for energy. Nat Nanotechnol 9:739-741

Marc G, McMillan W (1985) The virial theorem. Adv Chem Phys 58:209

Mics Z, Tielrooij K-J, Parvez K, Jensen S, Ivanov I, Feng X, M“ullen K, Bonn M, Turchinovich D (2015) Thermodynamic picture of ultrafast charge transport in graphene. Nat Commun 6:7655

Morpurgo A (2015) Ten years of nature physics: the abc of 2D materials. Nat Phys 11:625-626

Paggi M, Berardone I, Infuso A, Corrado M (2014) Fatigue degradation and electric recovery in silicon solar cells embedded in photovoltaic modules. Sci Rep 4:4506

Paggi M, Corrado M, Rodriguez MA (2013) A multi-physics and multi-scale numerical approach to microcracking and power-loss in photovoltaic modules. Compos Struct 95:630-638

Paggi M, Kajari-Schröder S, Eitner U (2011) Thermomechanical deformations in photovoltaic laminates. J Strain Anal Eng Des 46(8):772-782

Pei Q, Zhang Y, Shenoy V (2010) A molecular dynamics study of the mechanical properties of hydrogen functionalized graphene. Carbon 48:898-904

Peng Z, Lulu M, Feifei F, Zhi Z, Cheng P, Phillip EL, Zheng L, Yongji G, Jiangnan Z, Xingxiang Z, Pulickel MA, Ting Z, Jun L (2014) Fracture toughness of graphene. Nat Commun $5: 3782$

Plimpton S (1995) Fast parallel algorithms for short-range molecular dynamics. J Comput Phys 117:1-19

Pospischil A, Furchi M, Mueller T (2014) Solar-energy conversion and light emission in an atomic monolayer $\mathrm{p}-\mathrm{n}$ diode. Nat Nanotechnol 9:257-261

Quan L, Zhen T, Xueqian Z, Ranjan S, Liangliang D, Jianqiang G, Jiaguang H, Weili Z (2015) Active graphene-silicon hybrid diode for terahertz waves. Nat Commun 6:7082

Rodrigues G, Zelenovskiy P, Romanyuk K, Luchkin S, Kopelevich Y, Kholkin A (2015) Strong piezoelectricity in singlelayer graphene deposited on $\mathrm{sio}_{2}$ grating substrates. Nat Commun 6:7572

Santanu S, Elena B, Haddon RC (2012) Covalent chemistry in graphene electronics. Mater Today 15(6):276-285

Sarma D, Adam S, Hwang E, Rossi E (2011) Electronic transport in two dimensional graphene. Rev Mod Phys 83:407-470

Schröder SK, Kunze I, Köntges M (2012) Criticality of cracks in PV modules. Energy Procedia 27:658-663 
Schwierz F (2010) Graphene transistors. Nat Nanotechnol 5:487-496

Shenderova O, Brenner D, Omeltchenko A, Su X, Yang L (2000) Atomistic modeling of the fracture of polycrystalline diamond. Phys Rev B 61(6):3877-3888

Shin S, Choi H, Shin J, Bae D (2015) Strengthening behavior of few-layered graphene/ aluminum composites. Carbon $82: 143-151$

Son I, Park J, Kwon S, Park S, R"ummeli M, Bachmatiuk A, Song H, Ku J, Choi J, Choi J, Doo S-G, Chang H (2015) Silicon carbide-free graphene growth on silicon for lithium-ion battery with high volumetric energy density. Nat Commun 6:7393

Subramaniyan A, Sun C (2008) Continuum interpretation of virial stress in molecular simulations. Int J Solids Struct 45:4340-4346

Sun X-Y, Hu H, Cao C, Xua Y-J (2015) Anisotropic vacancydefect-induced fracture strength loss of graphene. RSC Adv 5:13623-13627

Swope W, Anderson H, Berens P, Wilson K (1982) A computer simulation method for the calculation of equilibrium constants for the formation of physical clusters of molecules: application to small water clusters. J Chem Phys 76:637

Tersoff J (1989) Modeling solid-state chemistry: interatomic potentials for multicomponent systems. Phys Rev B 39(8):5566-5568

Thomas S, Ajith K (2014) Molecular dynamics simulation of the thermo-mechanical properties of monolayer graphene sheet. Proc Mater Sci 5:489-498

Tiwary C, Javvaji B, Kumar C, Mahapatra D, Ozden S, Ajayan P, Chattopadhyay K (2015a) Chemical-free graphene by unzipping carbon nanotubes using cryo-milling. Carbon 217-224

Tiwary C, Vishnu D, Kole A, Brahmanandam J, Mahapatra D, Kumbhakar P, Chattopadhyay K (2015b) Stabilization of the high-temperature and high-pressure cubic phase of $\mathrm{ZnO}$ by temperature-controlled milling. J Mater Sci. doi:10.1007/ s10853-015-9394-1

Traversi F, Raillon C, Benameur S, Liu K, Khlybov S, Tosun M, Krasnozhon D, Kis A, Radenovic A (2014) Detecting the translocation of DNA through a nanopore using graphene nanoribbons. Nature Nanotechnol 8:939-945

Vadukumpully S, Paul J, Mahanta N, Valiyaveettil S (2011) Flexible conductive graphene/poly(vinyl chloride) composite thin films with high mechanical strength and thermal stability. Carbon 49:198-205
Volokh KY (2012) On the strength of graphene. J Appl Mech 79(6):064501-064501. doi:10.1115/1.4005582

Weiwei Z, Yanlei W, Zhangting W, Wenhui W, Kedong B, Zheng L, Juekuan Y, Yunfei C, Zhiping X, Zhenhua N (2015) Defect-engineered heat transport in graphene: a route to high efficient thermal rectification. Sci Rep 5:11962

Wen S, Yuqi Y, Jiangnan S, Hua C (2012) A graphene-based composite material noncovalently functionalized with a chemiluminescence reagent: synthesis and intrinsic chemiluminescence activity. Chem Commun 48:2894-2896

Xue T, Peng B, Xue M, Zhong X, Chiu C-Y, Yang S, Qu Y, Ruan L, Jiang S, Dubin S, Kaner R, Zink J, Meyerhoff M, Duan X, Huang Y (2014) Integration of molecular and enzymatic catalysts on graphene for biomimetic generation of antithrombotic species. Nat Commun 5:3200

Xuechao Y, Youde S, Tao L, Tao(Tom) W, Wang QJ (2015) Photocurrent generation in lateral graphene $\mathrm{p}-\mathrm{n}$ junction created by electron-beam irradiation. Sci Rep 5:12014

Ying-Yan Z, Qing-Xiang P, Yiu-Wing M, Yuan-Tong G (2014) Temperature and strain-rate dependent fracture strength of graphynes. J Phys D Appl Phys 47(42):425301

Zan L, Genlian F, Qiang G, Zhiqiang L, Yishi S, Di Z (2015) Synergistic strengthening effect of graphene-carbon nanotube hybrid structure in aluminum matrix composites. Carbon 95:419-427

Zhang H, Wu J, He J, Zhang Z (2012a) Effect of hole size on the fracture of graphene nanomesh. In: Proceeding of the 19th European conference on fracture, ISBN 978-5-905576-18-8

Zhang T, Li X, Kadkhodaei S, Gao H (2012b) Flaw insensitive fracture in nanocrystalline graphene. Nano Lett 12:46054610

Zhang Y, Zhao J, Wie N, Jiang J, Rabczuk T (2013) Effects of the dispersion of polymer wrapped two neighbouring single walled carbon nanotubes (SWNTs) on nanoengineering load transfer. Compos Part B Eng 45(1):1714-1721

Zhao H, Aluru N (2010) Temperature and strain-rate dependent fracture strength of graphene. J Appl Phys 108:064321

Zhao H, Min K, Aluru N (2009) Size and chirality dependent elastic properties of graphene nanoribbons under uniaxial tension. Nano Lett 9(8):3012-3015 